\title{
GotTLIEB GROUPS OF SPHERES
}

\author{
Marek Golasiński and Juno Mukai
}

\begin{abstract}
This paper takes up the systematic study of the Gottlieb groups $G_{n+k}\left(\mathbb{S}^{n}\right)$ of spheres for $k \leq 13$ by means of the classical homotopy theory methods. The groups $G_{n+k}\left(\mathbb{S}^{n}\right)$ for $k \leq 7$ and $k=10,12,13$ are fully determined. Partial results on $G_{n+k}\left(\mathbb{S}^{n}\right)$ for $k=8,9,11$ are presented as well. We also show that $\left[\iota_{n}, \eta_{n}^{2} \sigma_{n+2}\right]=0$ if $n=2^{i}-7$ for $i \geq 4$.
\end{abstract}

\section{Introduction}

The Gottlieb groups $G_{k}(X)$ of a pointed space $X$ have been defined by Gottlieb in [7] and [8]; first $G_{1}(X)$ and then $G_{k}(X)$ for all $k \geq 1$. The higher Gottlieb groups $G_{k}(X)$ are related in [8] and [9] to the existence of sectioning fibrations with fiber $X$. For instance, if $G_{k}(X)$ is trivial then there is a homotopy section for every fibration over the $(k+1)$-sphere $\mathbb{S}^{k+1}$, with fiber $X$.

This paper grew out of our attempt to develop techniques in calculating $G_{n+k}\left(\mathbb{S}^{n}\right)$ for $k \leq 13$ and any $n \geq 1$. The composition methods developed by Toda 31] are the main tools used in the paper. Our calculations also deeply depend on the results of [12, 14] and [19].

Section 1 serves as backgrounds to the rest of the paper. Write $\iota_{n}$ for the homotopy class of the identity map of $\mathbb{S}^{n}$. Then, the homomorphism

$$
P^{\prime}: \pi_{k}\left(\mathbb{S}^{n}\right) \longrightarrow \pi_{k+n-1}\left(\mathbb{S}^{n}\right)
$$

2000 Mathematics Subject Classification.Primary 55M35, 55Q52; Secondary 57S17. Key words and phrases: EHP sequence, fibration, Gottlieb group, rotation group, Stiefel manifold, Whitehead product.

The first author was partially supported by Akio Satō, Shinshu University 101501. The second author was partially supported by Grant-in-Aid for Scientific Research (No. 15540067 (c), (2)), Japan Society for the Promotion of Science and the Faculty of Mathematics and Computer Science in Toruń. 
defined by $P^{\prime}(\alpha)=\left[\iota_{n}, \alpha\right]$ for $\alpha \in \pi_{k}\left(\mathbb{S}^{n}\right)$ [10] leads to the formula $G_{k}\left(\mathbb{S}^{n}\right)=$ ker $P^{\prime}$, where $[-,-]$ terms the standard Whitehead product. So, our main task is to consult first [10, 11], [18, [19, 30] and [31] about the order of $\left[\iota_{n}, \alpha\right]$ and then to determine some Whitehead products in unsettled cases as well. In the light of Serre's result [29], the $p$-primary component of $G_{2 m+k}\left(\mathbb{S}^{2 m}\right)$ vanishes for any odd prime $p$, if $2 m \geq k+1$ (Proposition 1.4).

Let $E X$ be the suspension of a space $X$ and denote by $E: \pi_{k}(X) \rightarrow$ $\pi_{k+1}(E X)$ the suspension map. Write $\eta_{2} \in \pi_{3}\left(\mathbb{S}^{2}\right), \nu_{4} \in \pi_{7}\left(\mathbb{S}^{4}\right)$ and $\sigma_{8} \in$ $\pi_{15}\left(\mathbb{S}^{8}\right)$ for the Hopf maps, respectively. We set $\eta_{n}=E^{n-2} \eta_{2} \in \pi_{n+1}\left(\mathbb{S}^{n}\right)$ for $n \geq 2, \nu_{n}=E^{n-4} \nu_{4} \in \pi_{n+3}\left(\mathbb{S}^{n}\right)$ for $n \geq 4$ and $\sigma_{n}=E^{n-8} \sigma_{8} \in \pi_{n+7}\left(\mathbb{S}^{n}\right)$ for

$n \geq 8$. Write $\eta_{n}^{2}=\eta_{n} \circ \eta_{n+1}, \nu_{n}^{2}=\nu_{n} \circ \nu_{n+3}$ and $\sigma_{n}^{2}=\sigma_{n} \circ \sigma_{n+7}$. Section 2 is a description of $G_{n+k}\left(\mathbb{S}^{n}\right)$ for $k \leq 7$. To reach that for $G_{n+6}\left(\mathbb{S}^{n}\right)$, we make use of Theorem 2.2 partially extending the result of [15]: $\left[\iota_{n}, \nu_{n}^{2}\right]=0$ if and only if $n \equiv 4,5,7(\bmod 8), n=2^{i}-3$ or $n=2^{i}-5$ for $i \geq 4$; for the proof of which Section 3 and Section 4 are devoted.

Section 5 devotes to proving Mahowald's claim: $\left[\iota_{n}, \sigma_{n}\right] \neq 0$ for $n \equiv$ $7(\bmod 16) \geq 23$.

Section 6 takes up computations of $G_{n+k}\left(\mathbb{S}^{n}\right)$ for $k=10,12,13$ and partial ones of $G_{n+k}\left(\mathbb{S}^{n}\right)$ for $k=8,9,11$. In a repeated use of [19], we have found out the triviality of the Whitehead product [21]:

$$
\left[\iota_{n}, \eta_{n}^{2} \sigma_{n+2}\right]=0, \text { if } n=2^{i}-7(i \geq 4),
$$

which corrects thereby [19] for $n=2^{i}-7$.

The authors thank Professor M. Mimura for suggesting the problem and fruitful conversations, Professors H. Ishimoto, I. Madsen, M. Mahowald, Y. Nomura and N. Oda for helpful informations on the orders of the Whitehead products $\left[\iota_{n}, \nu_{n}^{2}\right]$ and $\left[\iota_{n}, \sigma_{n}\right]$.

The authors are also very grateful to Professor H. Toda for informing the order of the Whitehead product $\left[\iota_{2 n},\left[\iota_{2 n}, \alpha_{1}(2 n)\right]\right]$ for $n \geq 2$ (Proposition 1.5), where $\alpha_{1}(2 n)$ is a generator of the 3-primary component of $\pi_{2 n+3}\left(\mathbb{S}^{2 n}\right)$.

\section{Preliminaries on Gottlieb groups}

Throughout this paper, spaces, maps and homotopies are based. We use the standard terminology and notations from the homotopy theory, mainly from 31. We do not distinguish between a map and its homotopy class. 
Let $X$ be a connected space. The $k$-th Gottlieb group $G_{k}(X)$ of $X$ is the subgroup of the $k$-th homotopy group $\pi_{k}(X)$ consisting of all elements which can be represented by a map $f: \mathbb{S}^{k} \rightarrow X$ such that $f \vee \mathrm{id}_{X}: \mathbb{S}^{k} \vee X \rightarrow X$ extends (up to homotopy) to a map $F: \mathbb{S}^{k} \times X \rightarrow X$. Define $P_{k}(X)$ to be the set of elements of $\pi_{k}(X)$ whose Whitehead product with all elements of all homotopy groups is zero. It turns out that $P_{k}(X)$ forms a subgroup of $\pi_{k}(X)$ and, by [8, Proposition 2.3], $G_{k}(X) \subseteq P_{k}(X)$. Recall that $X$ is said to be a $G$-space (resp. $W$-space) if $\pi_{k}(X)=G_{k}(X)\left(\right.$ resp. $\left.\pi_{k}(X)=P_{k}(X)\right)$ for all $k$.

Given $\alpha \in \pi_{k}\left(\mathbb{S}^{n}\right)$ for $k \geq 1$, we deduce that $\alpha \in G_{k}\left(\mathbb{S}^{n}\right)$ if and only if $\left[\iota_{n}, \alpha\right]=0$. In other words, consider the map

$$
P^{\prime}: \pi_{k}\left(\mathbb{S}^{n}\right) \longrightarrow \pi_{k+n-1}\left(\mathbb{S}^{n}\right)
$$

defined by $P^{\prime}(\alpha)=\left[\iota_{n}, \alpha\right]$ for $\alpha \in \pi_{k}\left(\mathbb{S}^{n}\right)$. Then, this leads to the formula

$$
G_{k}\left(\mathbb{S}^{n}\right)=\operatorname{ker} P^{\prime} .
$$

Write now $\sharp$ for the order of a group or its any element. Then, from the above interpretation of Gottlieb groups of spheres, we obtain

Lemma 1.1 If $\pi_{k}\left(\mathbb{S}^{n}\right)$ is a cyclic group for some $k \geq 1$ with a generator $\alpha$ then $G_{k}\left(\mathbb{S}^{n}\right)=\left(\sharp\left[\iota_{n}, \alpha\right]\right) \pi_{k}\left(\mathbb{S}^{n}\right)$.

Since $\mathbb{S}^{n}$ is an H-space for $n=3,7$, we have

$$
G_{k}\left(\mathbb{S}^{n}\right)=\pi_{k}\left(\mathbb{S}^{n}\right) \text { for } k \geq 1 \text {, if } n=3,7 .
$$

We recall the following result from [11] and [37] needed in the sequel.

Lemma 1.2 (1) If $\xi \in \pi_{m}(X), \eta \in \pi_{n}(X), \alpha \in \pi_{k}\left(\mathbb{S}^{m}\right), \beta \in \pi_{l}\left(\mathbb{S}^{n}\right)$ and if $[\xi, \eta]=0$ then $[\xi \circ \alpha, \eta \circ \beta]=0$.

(2) Let $\alpha \in \pi_{k+1}(X), \beta \in \pi_{l+1}(X), \gamma \in \pi_{m}\left(\mathbb{S}^{k}\right)$ and $\delta \in \pi_{n}\left(\mathbb{S}^{l}\right)$.

Then $[\alpha \circ E \gamma, \beta \circ E \delta]=[\alpha, \beta] \circ E(\gamma \wedge \delta)$.

(3) If $\alpha \in \pi_{k}\left(\mathbb{S}^{2}\right)$ and $\beta \in \pi_{l}\left(\mathbb{S}^{2}\right)$ then $[\alpha, \beta]=0$ unless $k=l=2$.

(4) $[\beta, \alpha]=(-1)^{(k+1)(l+1)}[\alpha, \beta]$ for $\alpha \in \pi_{k+1}(X)$ and $\beta \in \pi_{l+1}(X)$.

In particular, $2[\alpha, \alpha]=0$ for $\alpha \in \pi_{n}(X)$ if $n$ is odd.

(5) If $\alpha_{1}, \alpha_{2} \in \pi_{p+1}(X), \beta \in \pi_{q+1}(X)$ and $p \geq 1$, then $\left[\alpha_{1}+\alpha_{2}, \beta\right]=$ $\left[\alpha_{1}, \beta\right]+\left[\alpha_{2}, \beta\right]$ and $\left[\beta, \alpha_{1}+\alpha_{2}\right]=\left[\beta, \alpha_{1}\right]+\left[\beta, \alpha_{2}\right]$.

(6) $E[\alpha, \beta]=0$ for $\alpha \in \pi_{k}(X)$ and $\beta \in \pi_{l}(X)$.

(7) $3[\alpha,[\alpha, \alpha]]=0$ for $\alpha \in \pi_{n+1}(X)$. 
Let $G_{k}(X ; p)$ and $\pi_{k}(X ; p)$ be the $p$-primary components of $G_{k}(X)$ and $\pi_{k}(X)$ for a prime $p$, respectively. But for $X=\mathbb{S}^{n}$, recall the notation from 31:

$$
\pi_{k}^{n}= \begin{cases}\pi_{n}\left(\mathbb{S}^{n}\right), & \text { if } k=n ; \\ E^{-1} \pi_{2 n}\left(\mathbb{S}^{n+1} ; 2\right), & \text { if } k=2 n-1 \\ \pi_{k}\left(\mathbb{S}^{n} ; 2\right), & \text { if } k \neq n, 2 n-1 .\end{cases}
$$

As it is well-known, $\left[\iota_{n}, \iota_{n}\right]=0$ if and only if $n=1,3,7$ and $\sharp\left[\iota_{n}, \iota_{n}\right]=2$ for $n$ odd and $n \neq 1,3,7$, and it is infinite provided $n$ is even. Thus, we have reproved the result [8] that $G_{n}\left(\mathbb{S}^{n}\right)=\pi_{n}\left(\mathbb{S}^{n}\right) \cong \mathbb{Z}$ for $n=1,3,7$, $G_{n}\left(\mathbb{S}^{n}\right)=2 \pi_{n}\left(\mathbb{S}^{n}\right) \cong 2 \mathbb{Z}$ for $n$ odd and $n \neq 1,3,7$, and $G_{n}\left(\mathbb{S}^{n}\right)=0$ for $n$ even, where $\mathbb{Z}$ denotes the additive group of integers. It is easily obtained that $G_{k}\left(\mathbb{S}^{n}\right)=P_{k}\left(\mathbb{S}^{n}\right)$ for all $k, n$ [16, Theorem I.9]. In other words, on the level of spheres the class of $G$-spaces coincides with that of $W$-spaces.

Let now $n$ be odd. Then, by Lemma 1.2 (4), (5) and $(7),\left[\iota_{n},\left[\iota_{n}, \iota_{n}\right]\right]=0$. Furthermore, by Lemma 1.2. (1), (4) and (5), $\left[2 \iota_{n}, \iota_{n}\right]=0$ implies that $\left[\iota_{n}, 2 \alpha\right]=0$ for any $\alpha \in \pi_{k}\left(\mathbb{S}^{n}\right)$, that is, $2 \pi_{k}\left(\mathbb{S}^{n}\right) \subset G_{k}\left(\mathbb{S}^{n}\right)$ and thus $G_{k}\left(\mathbb{S}^{n} ; p\right)=$ $\pi_{k}\left(\mathbb{S}^{n} ; p\right)$ for any odd prime $p$. In the light of [17, we also know

$$
\sharp\left[\iota_{2 n},\left[\iota_{2 n}, \iota_{2 n}\right]\right]=3 \text {, if } n \geq 2 .
$$

Whence, Lemma 1.2 and (1.1) yield the results proved partially in [6].

Corollary 1.3. (1) $3\left[\iota_{n}, \iota_{n}\right] \in G_{2 n-1}\left(\mathbb{S}^{n}\right)$. In particular, $a\left[\iota_{n}, \iota_{n}\right] \in$ $G_{2 n-1}\left(\mathbb{S}^{n}\right)$ for $n$ even if and only if $a \equiv 0(\bmod 3)$.

(2) If $k \geq 3$ then $G_{k}\left(\mathbb{S}^{2}\right)=\pi_{k}\left(\mathbb{S}^{2}\right)$.

(3) If $n$ is odd and $n \neq 1,3,7$ then $2 \pi_{k}\left(\mathbb{S}^{n}\right) \subset G_{k}\left(\mathbb{S}^{n}\right)$. In particular, $G_{k}\left(\mathbb{S}^{n} ; p\right)=\pi_{k}\left(\mathbb{S}^{n} ; p\right)$ for any odd prime $p$ and $k \geq 1$.

(4) $G_{k}\left(\mathbb{S}^{n}\right)=\pi_{k}\left(\mathbb{S}^{n}\right)$ provided that $E: \pi_{k+n-1}\left(\mathbb{S}^{n}\right) \rightarrow \pi_{k+n}\left(\mathbb{S}^{n+1}\right)$ is a monomorphism.

We note that $P^{\prime}$ and the homomorphism

$$
P: \pi_{k+n+1}\left(\mathbb{S}^{2 n+1}\right) \longrightarrow \pi_{k+n-1}\left(\mathbb{S}^{n}\right)(k \leq 2 n-2)
$$

in the EHP sequence are related as follows:

$$
P^{\prime}=P \circ E^{n+1} \text { for } k \leq 2 n-2 .
$$


Let $S O(n)$ be the rotation group and $J: \pi_{k}(S O(n)) \rightarrow \pi_{n+k}\left(\mathbb{S}^{n}\right)$ be the $J$-homomorphism and $\Delta: \pi_{k}\left(\mathbb{S}^{n}\right) \rightarrow \pi_{k-1}(S O(n))$ the connecting map associated with the fibration $S O(n+1) \stackrel{S O(n)}{\longrightarrow} \mathbb{S}^{n}$. We recall

$$
P^{\prime}=J \circ \Delta
$$

and so,

$$
\operatorname{ker}\left\{\Delta: \pi_{k}\left(\mathbb{S}^{n}\right) \rightarrow \pi_{k-1}(S O(n))\right\} \subset G_{k}\left(\mathbb{S}^{n}\right) .
$$

In virtue of [29, Chapter IV] (31, (13.1)]), Serre's isomorphism

$$
\pi_{i-1}\left(\mathbb{S}^{2 m-1} ; p\right) \oplus \pi_{i}\left(\mathbb{S}^{4 m-1} ; p\right) \cong \pi_{i}\left(\mathbb{S}^{2 m} ; p\right)
$$

is given by the correspondence $(\alpha, \beta) \mapsto E \alpha+\left[\iota_{2 m}, \iota_{2 m}\right] \circ \beta$.

We show

Proposition 1.4. Let $p$ be an odd prime and $n$ even.

(1) If $n \geq k+1$ then $G_{n+k}\left(\mathbb{S}^{n} ; p\right)=0$.

(2) Suppose that $\pi_{n+k}\left(\mathbb{S}^{n} ; p\right)$ is cyclic with a generator $\theta, \pi_{n+k}\left(\mathbb{S}^{n} ; p\right)=$ $E \pi_{n+k-1}\left(\mathbb{S}^{n-1} ; p\right)$ and that $E^{n-1}: \pi_{n+k}\left(\mathbb{S}^{n} ; p\right) \rightarrow \pi_{2 n+k-1}\left(\mathbb{S}^{2 n-1} ; p\right)$ is an epimorphism. Then, $G_{n+k}\left(\mathbb{S}^{n} ; p\right)=\left\{\sharp\left(\operatorname{ker} E^{n-1}\right) \theta\right\}$.

Proof. By the Freudenthal suspension theorem, $E: \pi_{n+k}\left(\mathbb{S}^{n}\right) \rightarrow \pi_{n+k+1}\left(\mathbb{S}^{n+1}\right)$ is an epimorphism, if $n=k+1$ and an isomorphism, if $n \geq k+2$. For the case $n=k+1$, by the EHP sequence

$$
\pi_{2 n-2}\left(\mathbb{S}^{n-1}\right) \stackrel{E}{\longrightarrow} \pi_{2 n-1}\left(\mathbb{S}^{n}\right) \stackrel{H}{\longrightarrow} \pi_{2 n-1}\left(\mathbb{S}^{2 n-1}\right)
$$

we get

$$
\pi_{2 n-1}\left(\mathbb{S}^{n}\right)=E \pi_{2 n-2}\left(\mathbb{S}^{n-1}\right) \oplus \mathbb{Z}\{\alpha\},
$$

where $\alpha=\eta_{2}, \nu_{4}, \sigma_{8}$ according as $n=2,4,8$ and $\alpha=\left[\iota_{n}, \iota_{n}\right]$ for $n$ otherwise. So, by the EHP sequence

$$
\pi_{2 n+1}\left(\mathbb{S}^{2 n+1}\right) \stackrel{P}{\longrightarrow} \pi_{2 n-1}\left(\mathbb{S}^{n}\right) \stackrel{E}{\longrightarrow} \pi_{2 n}\left(\mathbb{S}^{n+1}\right)
$$

and by the Freudenthal suspension theorem,

$$
\sharp \beta=\sharp\left(E^{m} \beta\right) \text { for any } \beta \in \pi_{n+k}\left(\mathbb{S}^{n} ; p\right) \text { and } m \geq 1 \text {. }
$$


Hence, by Lemma 1.2.(2) and (1.4), we obtain

$$
\sharp \beta=\sharp\left[\iota_{n}, \beta\right] .
$$

This leads to (1).

(2) is easily obtained from (1.4) and the proof is complete.

The notation $\pi_{n+m}\left(\mathbb{S}^{n}\right)=\left\{\alpha_{n}\right\}(\{\alpha(n)\}$, resp. $)$ means that there exist some $k \geq 1$ and an element $\alpha_{k}(\alpha(k)$, resp. $) \in \pi_{k+m}\left(\mathbb{S}^{k}\right)$ satisfying $\alpha_{n}=$ $E^{n-k} \alpha_{k}\left(\alpha(n)=E^{n-k} \alpha(k)\right.$, resp. $)$ for $n \geq k$. For the $p$-primary component with any prime $p$, the notation is available.

Hereafter, we omit the reference [31] unless otherwise stated. Now, we know that $\pi_{n+3}\left(\mathbb{S}^{n} ; 3\right)=\left\{\alpha_{1}(n)\right\} \cong \mathbb{Z}_{3}$ and $\pi_{n+7}\left(\mathbb{S}^{n} ; 3\right)=\left\{\alpha_{2}(n)\right\} \cong \mathbb{Z}_{3}$ for $n \geq 3$. Write $\{-,-,-\}_{n}$ for the Toda bracket, where $n \geq 0$ and $\{-,-,-\}=\{-,-,-\}_{0}$. We recall that there exists the element $\beta_{1}(5) \in$ $\pi_{15}\left(\mathbb{S}^{5}\right)$ satisfying $\beta_{1}(5) \in\left\{\alpha_{1}(5), \alpha_{1}(8), \alpha_{1}(11)\right\}_{1}, 3 \beta_{1}(5)=-\alpha_{1}(5) \alpha_{2}(8)$ and that $\pi_{n+10}\left(\mathbb{S}^{n} ; 3\right)=\left\{\beta_{1}(n)\right\} \cong \mathbb{Z}_{9}$ for $n=5,6$ and $\cong \mathbb{Z}_{3}$ for $n \geq 7$.

Let $\Omega^{2} \mathbb{S}^{2 m+1}=\Omega\left(\Omega \mathbb{S}^{2 m+1}\right)$ be the double loop space of $\mathbb{S}^{2 m+1}$ and $Q_{2}^{2 m-1}=$ $\Omega\left(\Omega^{2} \mathbb{S}^{2 m+1}, \mathbb{S}^{2 m-1}\right)$ the homotopy fiber of the canonical inclusion (the double suspension map) $i: \mathbb{S}^{2 m-1} \rightarrow \Omega^{2} \mathbb{S}^{2 m+1}$. Then, the following result and its proof have been shown by Toda [35].

Proposition 1.5. Let $n$ be even and $n \geq 4$. Then $\left[\iota_{n},\left[\iota_{n}, \alpha_{1}(n)\right]\right] \neq 0$ if and only if $n \neq 4$ and $n \equiv 1(\bmod 3)$.

Proof. It is well-known that $\left[\iota_{n},\left[\iota_{n}, \iota_{n}\right]\right] \in E \pi_{3 n-3}\left(\mathbb{S}^{n-1}\right)$. So, by the (mod 3) EHP sequence [34. (2.1.3), Proposition 2.1], we have

$\left[\iota_{n},\left[\iota_{n}, \iota_{n}\right]\right]= \pm E P(i(n-1))$, where $i(n-1)$ is a generator of $\pi_{3 n-3}\left(Q_{2}^{n-1} ; 3\right)$.

By the naturality [34, (2.1.5)], we obtain $\left[\iota_{n},\left[\iota_{n}, \alpha_{1}(n)\right]\right]= \pm E P\left(a_{1}(n-1)\right)$, where $a_{1}(n-1)=i(n-1) \alpha_{1}(3 n-3) \in \pi_{3 n}\left(Q_{2}^{n-1} ; 3\right)$. By [34, Proposition 4.4], $\left(\frac{n}{2}+1\right) a_{1}(n-1)=H P(i(n+1))$. So, $P\left(a_{1}(n-1)\right)= \pm P H P(i(n+1))=0$ if $n \not \equiv 1(\bmod 3)$. For the case $n=4$, the assertion is trivial.

Next, assume that $n \neq 4$ and $n \equiv 1(\bmod 3)$. Then, by [33, Theorem 10.3], there exists an element $v \in \pi_{3 n-4}\left(\mathbb{S}^{n-3}\right)$ satisfying $H(v)=b(n-5)$ and $E^{2} v=P\left(a_{1}(n-1)\right)$, where $b(n-5)=i(n-5) \beta_{1}(3 n-15)$. Furthermore, by [33. Proposition 5.3.(ii)], we obtain $P\left(a_{2}(n-3)\right)=3 v$, where $a_{2}(n-3)=i(n-$ $3) \alpha_{2}(3 n-9)$. So, by the $(\bmod 3)$ EHP sequence, we have $P\left(a_{1}(n-1)\right) \neq 0$. This implies the sufficient condition and completes the proof. 


\section{Gottlieb groups of spheres with stems for $k \leq 7$}

According to [10], 11], 15], 18, 30] and [31], we know the following results:

$$
\begin{aligned}
& {\left[\iota_{n}, \eta_{n}\right]=0 \text { if and only if } n \equiv 3(\bmod 4) \text { or } n=2,6 ;} \\
& {\left[\iota_{n}, \eta_{n}^{2}\right]=0 \text { if and only if } n \equiv 2,3(\bmod 4) \text { or } n=5 .}
\end{aligned}
$$

Hence, Lemma 1.1 completely determines $G_{n+k}\left(\mathbb{S}^{n}\right)$ for $k=1,2$.

We recall that $\pi_{6}^{3}=\left\{\nu^{\prime}\right\} \cong \mathbb{Z}_{4}$. Write $\omega$ for a generator of the $J$-image $J \pi_{3}(S O(3))=\pi_{6}\left(\mathbb{S}^{3}\right) \cong \mathbb{Z}_{12}$ satisfying $\omega=\nu^{\prime}+\alpha_{1}(3)$. We recall the relation $\pm\left[\iota_{4}, \iota_{4}\right]=2 \nu_{4}-\Sigma \nu^{\prime}$. By abuse of notation, $\nu_{n}$ represents a generator of $\pi_{n+3}^{n}$ and $\pi_{n+3}\left(\mathbb{S}^{n}\right)$ for $n \geq 4$, respectively. Then, $\pi_{7}\left(\mathbb{S}^{4}\right)=\left\{\nu_{4}, E \omega\right\} \cong \mathbb{Z} \oplus \mathbb{Z}_{12}$, $\pi_{n+3}\left(\mathbb{S}^{n}\right)=\left\{\nu_{n}\right\} \cong \mathbb{Z}_{24}$ for $n \geq 5$. By [2], $\left[\iota_{4}, \nu_{4}\right]=2 \nu_{4}^{2}$. In the light of Lemma 1.2. (2) and the relation $\nu^{\prime} \nu_{6}=0$, we obtain

$$
\left[\iota_{4}, E \nu^{\prime}\right]=\left[\iota_{4}, \iota_{4}\right] \circ E\left(\iota_{3} \wedge \nu^{\prime}\right)=\left(2 \nu_{4}-E \nu^{\prime}\right) \circ 2 \nu_{7}=4 \nu_{4}^{2} .
$$

So, we have $2 E \nu^{\prime}=\eta_{4}^{3} \in G_{7}\left(\mathbb{S}^{4}\right)$. Consequently, by Corollary 1.3. (1) and Proposition 1.4.

$$
G_{7}\left(\mathbb{S}^{4}\right)=\left\{3\left[\iota_{4}, \iota_{4}\right], 2 E \nu^{\prime}\right\} \cong 3 \mathbb{Z} \oplus \mathbb{Z}_{2} .
$$

In the light of [15], 18, 30, 31, Corollary 1.3. (3) and Proposition 1.4, we know the following:

$$
\sharp\left[\iota_{n}, \nu_{n}\right]= \begin{cases}1, & \text { if } n \equiv 7(\bmod 8), n=2^{i}-3 \text { for } i \geq 3 ; \\ 2, & \text { if } n \equiv 1,3,5(\bmod 8) \geq 9 \text { and } n \neq 2^{i}-3 ; \\ 12, & \text { if } n \equiv 2(\bmod 4) \geq 6, n=12 \\ 24, & \text { if } n \equiv 0(\bmod 4) \geq 8 \text { unless } 12 .\end{cases}
$$

Thus, Lemma 1.1 leads to a complete description of $G_{n+3}\left(\mathbb{S}^{n}\right)$.

Now, we recall the following relations:

$$
\eta_{n} \nu_{n+1}=0 \text { for } n \geq 5 \text { and } \nu_{n} \eta_{n+3}=0 \text { for } n \geq 6 .
$$

By the relation $\nu^{\prime} \eta_{6}=\eta_{3} \nu_{4}$, we have $\left[\iota_{4}, \nu_{4} \eta_{7}\right]=\left[\iota_{4},\left(E \nu^{\prime}\right) \eta_{7}\right]=\left[\iota_{5}, \nu_{5} \eta_{8}\right]=0$. Hence, by the group structures of $\pi_{n+k}\left(\mathbb{S}^{n}\right)$ for $k=4,5$, we get 
Proposition 2.1. $\quad G_{n+4}\left(\mathbb{S}^{n}\right)=\pi_{n+4}\left(\mathbb{S}^{n}\right) ; G_{n+5}\left(\mathbb{S}^{n}\right)=\pi_{n+5}\left(\mathbb{S}^{n}\right)$ unless $n=$ 6 and $G_{11}\left(\mathbb{S}^{6}\right)=3 \pi_{11}\left(\mathbb{S}^{6}\right) \cong 3 \mathbb{Z}$.

In the next two sections, we will prove the following result partially extending that of [15, Theorem 1.3].

Theorem 2.2. $\left[\iota_{n}, \nu_{n}^{2}\right]=0$ if and only if $n \equiv 4,5,7(\bmod 8), n=2^{i}-3$ or $n=2^{i}-5$ for $i \geq 4$.

We recall that $\pi_{10}\left(\mathbb{S}^{4}\right)=\left\{\nu_{4}^{2}, \alpha_{1}(4) \alpha_{1}(7), \nu_{4} \alpha_{1}(7)\right\} \cong \mathbb{Z}_{8} \oplus\left(\mathbb{Z}_{3}\right)^{2}$. By [2] and the relation $\alpha_{1}(7) \alpha_{1}(10)=0$, we get that $\left[\iota_{4}, \nu_{4} \alpha_{1}(7)\right]=\left[\iota_{4}, \alpha_{1}(4) \alpha_{1}(7)\right]=$ $\left[\iota_{4}, \iota_{4}\right]\left(\alpha_{1}(7) \alpha_{1}(10)\right)=0$. Recall also that $\pi_{12}^{5}=\left\{\sigma^{\prime \prime \prime}\right\} \cong \mathbb{Z}_{2}, \pi_{13}^{6}=\left\{\sigma^{\prime \prime}\right\} \cong \mathbb{Z}_{4}$, $\pi_{14}^{7}=\left\{\sigma^{\prime}\right\} \cong \mathbb{Z}_{8}$, where $E \sigma^{\prime \prime \prime}=2 \sigma^{\prime \prime}, E \sigma^{\prime \prime}=2 \sigma^{\prime}$ and $E^{2} \sigma^{\prime}=2 \sigma_{9}$. By [2] and 31, we obtain

$$
\left[\iota_{5}, \sigma^{\prime \prime \prime}\right]=\left[\iota_{5}, \iota_{5}\right] \circ E^{4} \sigma^{\prime \prime \prime}=0,\left[\iota_{6}, \sigma^{\prime \prime}\right]=\left[\iota_{6}, \iota_{6}\right] \circ E^{5} \sigma^{\prime \prime}=4\left(\left[\iota_{6}, \iota_{6}\right] \circ \sigma_{11}\right)
$$

and $2\left[\iota_{6}, \sigma^{\prime \prime}\right] \neq 0$. We recall the relation $\pm\left[\iota_{8}, \iota_{8}\right]=2 \sigma_{8}-E \sigma^{\prime}$. In $\pi_{22}^{8}=$ $\mathbb{Z}_{16}\left\{\sigma_{8}^{2}\right\} \oplus \mathbb{Z}_{8}\left\{\left(E \sigma^{\prime}\right) \sigma_{15}\right\} \oplus \mathbb{Z}_{4}\left\{\kappa_{8}\right\}$, we have $\left[\iota_{8}, E \sigma^{\prime}\right]=2\left[\iota_{8}, \iota_{8}\right] \sigma_{15}= \pm 2\left(2 \sigma_{8}^{2}-\right.$ $\left.\left(E \sigma^{\prime}\right) \sigma_{15}\right)$ and in view of [2], we obtain $\left[\iota_{8}, \sigma_{8}\right]=\left[\iota_{8}, \iota_{8}\right] \circ \sigma_{15}= \pm\left(2 \sigma_{8}^{2}-\right.$ $\left.\left(E \sigma^{\prime}\right) \sigma_{15}\right)$. We know that $\pi_{n+7}\left(\mathbb{S}^{n} ; 5\right)=\left\{\alpha_{1}^{\prime}(n)\right\} \cong \mathbb{Z}_{5}$ for $n \geq 3$. Thus, by Corollary 1.3, Proposition 1.4 and Theorem 2.2. we obtain

Proposition 2.3. $\quad G_{n+6}\left(\mathbb{S}^{n}\right)=\pi_{n+6}\left(\mathbb{S}^{n}\right)$ if $n \equiv 4,5,7(\bmod 8), n=2^{i}-3$ or $n=2^{i}-5$ and $G_{n+6}\left(\mathbb{S}^{n}\right)=0$ otherwise.

Furthermore, $G_{n+7}\left(\mathbb{S}^{n}\right)=0$ if $n=4,6, G_{12}\left(\mathbb{S}^{5}\right)=\pi_{12}\left(\mathbb{S}^{5}\right), G_{15}\left(\mathbb{S}^{8}\right)=$ $\left\{3\left[\iota_{8}, \iota_{8}\right], 4 E \sigma^{\prime}\right\} \cong 3 \mathbb{Z} \oplus \mathbb{Z}_{2}$.

Let $H: \pi_{k}\left(\mathbb{S}^{n}\right) \rightarrow \pi_{k}\left(\mathbb{S}^{2 n-1}\right)$ be the Hopf homomorphism. Then, by [1] and [27, Proposition 4.5], there exists an element $\gamma \in \pi_{2 n-8}^{n-7}$ satisfying

$$
\left[\iota_{n}, \iota_{n}\right]=E^{7} \gamma \text { and } H \gamma=\sigma_{2 n-15}, \text { if } n \equiv 7(\bmod 16) \geq 23 .
$$

According to Mahowald [21] and (2.4), we obtain

Theorem 2.4. $\left[\iota_{n}, \sigma_{n}\right] \neq 0$, if $n \equiv 7(\bmod 16) \geq 23$. It desuspends seven dimensions whose Hopf invariant is $\sigma_{2 n-15}^{2}$. 
By abuse of notation, $\sigma_{n}$ represents a generator of $\pi_{n+7}^{n}$ and $\pi_{n+7}\left(\mathbb{S}^{n}\right)$ for $n \geq 8$, respectively. Combining the results of [18, 19, 31, Corollary 1.3. (3), Proposition 1.4 and Theorem 2.4 .

$$
\sharp\left[\iota_{n}, \sigma_{n}\right]= \begin{cases}240, & \text { if } n \text { is even and } n \geq 10 \\ 2, & \text { if } n \text { is odd and } n \geq 9 \text { unless } n=11 \\ & \text { or } n \equiv 15(\bmod 16) \\ 1, & \text { if } n=11 \text { or } n \equiv 15(\bmod 16) .\end{cases}
$$

Whence, by means of Lemma 1.1, the groups $G_{n+7}\left(\mathbb{S}^{n}\right)$ for $n \geq 9$ have been fully described as well.

\section{Proof of Theorem 2.2, part I}

Denote by $i_{n}(\mathbb{R}): S O(n-1) \hookrightarrow S O(n)$ and $p_{n}(\mathbb{R}): S O(n) \rightarrow \mathbb{S}^{n-1}$ the inclusion and projection maps, respectively. Hereafter, we use the following exact sequence induced from the fibration $S O(n+1) \stackrel{S O(n)}{\longrightarrow} \mathbb{S}^{n}$ :

$$
\left(\mathcal{S O}_{k}^{n}\right) \quad \pi_{k+1}\left(\mathbb{S}^{n}\right) \stackrel{\Delta}{\longrightarrow} \pi_{k}(S O(n)) \stackrel{i_{*}}{\longrightarrow} \pi_{k}(S O(n+1)) \stackrel{p_{*}}{\longrightarrow} \pi_{k}\left(\mathbb{S}^{n}\right) \longrightarrow \cdots,
$$

where $i=i_{n+1}(\mathbb{R})$ and $p=p_{n+1}(\mathbb{R})$.

Since $S O(n) \cong S O(n-1) \times \mathbb{S}^{n-1}$ for $n=4,8$, we get that

$$
\Delta \pi_{k+1}\left(\mathbb{S}^{n}\right)=0 \text {, if } n=3,7 .
$$

By the exact sequence $\left(\mathcal{S O}_{n}^{n}\right)$ and the fact that $\pi_{n}(S O(n)) \cong \mathbb{Z}$ for $n \equiv$ $3(\bmod 4)[14$, we have

$$
\Delta \eta_{n}=0, \text { if } n \equiv 3(\bmod 4) .
$$

We recall the formula [14, Lemma 1]

$$
\Delta(\alpha \circ E \beta)=\Delta \alpha \circ \beta .
$$

By (3.2) and (3.3),

$$
\Delta\left(\eta_{n}^{2}\right)=0, \text { if } n \equiv 3(\bmod 4) .
$$

Denote by $V_{n, k}$ the Stiefel manifold consisting of $k$-frames in $\mathbb{R}^{n}$ for $k \leq n-1$. Then, we show 
Lemma 3.1. (1) $\Delta\left(\nu_{n}^{2}\right)=0$, if $n \equiv 5(\bmod 8)$;

(2) $\Delta\left(\nu_{4 n}^{2}\right)=0$, if $n$ is odd.

Proof. Since $\pi_{7}(S O(5)) \cong \mathbb{Z}\left[14, \Delta: \pi_{8}\left(\mathbb{S}^{5}\right) \rightarrow \pi_{7}(S O(5))\right.$ is trivial and $\Delta \nu_{5}=0$. So, by $(3.3), \Delta\left(\nu_{5}^{2}\right)=0$. Let now $n \equiv 5(\bmod 8) \geq 13$. We consider the exact sequence $\left(\mathcal{S O}_{n+5}^{n}\right)$ :

$$
\pi_{n+6}\left(\mathbb{S}^{n}\right) \stackrel{\Delta}{\rightarrow} \pi_{n+5}(S O(n)) \stackrel{i_{*}}{\rightarrow} \pi_{n+5}(S O(n+1)) \rightarrow 0 .
$$

By [4], we obtain

$$
\pi_{n+5}(S O(n)) \cong \pi_{n+5}(S O) \oplus \pi_{n+6}\left(V_{n+8,8}\right) .
$$

In the light of [12], $\pi_{n+6}\left(V_{n+8,8}\right) \cong \mathbb{Z}_{8}$ and by [5], $\pi_{n+5}(S O)=0$. So, $\pi_{n+5}(S O(n)) \cong \mathbb{Z}_{8}$. By [14], $\pi_{n+5}(S O(n+1)) \cong \mathbb{Z}_{8}$. From the fact that $\pi_{n+6}\left(\mathbb{S}^{n}\right)=\left\{\nu_{n}^{2}\right\} \cong \mathbb{Z}_{2}$, we obtain $\Delta\left(\nu_{n}^{2}\right)=0$, and hence $(1)$ follows.

We obtain $\pi_{9}(S O(4)) \cong \pi_{9}(S O(3)) \oplus \pi_{9}\left(\mathbb{S}^{3}\right) \cong\left(\mathbb{Z}_{3}\right)^{2}$, and so $\Delta\left(\nu_{4}^{2}\right)=0$. Let now $n \geq 3$. Then, we consider the exact sequence $\left(\mathcal{S O}_{4 n+5}^{4 n}\right)$ :

$$
\pi_{4 n+6}\left(\mathbb{S}^{4 n}\right) \stackrel{\Delta}{\rightarrow} \pi_{4 n+5}(S O(4 n)) \stackrel{i_{*}}{\rightarrow} \pi_{4 n+5}(S O(4 n+1)) \rightarrow 0 .
$$

By [14], $\pi_{4 n+5}(S O(4 n+1)) \cong \mathbb{Z}_{2}$. By [13], $i_{13}(\mathbb{R})_{*}: \pi_{17}(S O(12)) \rightarrow \pi_{17}(S O(13)) \cong$ $\mathbb{Z}_{2}$ is an isomorphism, and hence $\Delta\left(\nu_{12}^{2}\right)=0$. Let $n$ be odd and $n \geq 5$. In the light of [4,

$$
\pi_{4 n+5}(S O(4 n)) \cong \pi_{4 n+5}(S O) \oplus \pi_{4 n+6}\left(V_{4 n+8,8}\right), \text { if } n \geq 4
$$

By means of [5] and [12], $\pi_{4 n+5}(S O) \cong \mathbb{Z}_{2}$ and $\pi_{4 n+6}\left(V_{4 n+8,8}\right)=0$. Hence, we obtain $\Delta\left(\nu_{4 n}^{2}\right)=0$ if $n$ is odd with $n \geq 5$. This leads to (2) and completes the proof.

[15. Theorem 1.3] suggests the non-triviality of $\left[\iota_{n}, \nu_{n}^{2}\right]$ for $n \equiv 0,1,2$, $3,6(\bmod 8) \geq 6$ and $[25$, Proposition 3.4 ] gives an explicit proof of its nontriviality for $n \equiv 2(\bmod 4) \geq 6$.

By Lemma 1.2. (1) and (2.3), we have $\left[\iota_{n}, \nu_{n}^{2}\right]=0$ if $n \equiv 7(\bmod 8)$ or $n=2^{i}-3$ for $i \geq 3$. In virtue of Lemma 3.1 and by (1.2), we get that

$$
\left[\iota_{n}, \nu_{n}^{2}\right]=0, \text { if } n \equiv 5(\bmod 8)
$$

and

$$
\left[\iota_{n}, \nu_{n}^{2}\right]=0, \text { if } n \equiv 4(\bmod 8)
$$


Given elements $\alpha \in \pi_{n+k}\left(\mathbb{S}^{n}\right)$ and $\beta \in \pi_{n+k}(S O(n+1))$ satisfying $p_{n+1}(\mathbb{R}) \beta=$ $\alpha$, then $\beta$ is called a lift of $\alpha$ and we write

$$
\beta=[\alpha]
$$

For $k \leq n-1$, set $i_{k, n}=i_{n}(\mathbb{R}) \circ \cdots \circ i_{k+1}(\mathbb{R})$.

Let now $n \equiv 0(\bmod 4) \geq 8$. By [4, [5] and [12], $\pi_{2 n+3}(S O(2 n-2)) \cong$ $\mathbb{Z} \oplus \mathbb{Z}_{4}$. In the exact sequence $\left(\mathcal{S O}_{2 n+3}^{2 n-3}\right), p_{2 n-2}(\mathbb{R})_{*}: \pi_{2 n+3}(S O(2 n-2)) \rightarrow$ $\pi_{2 n+3}\left(\mathbb{S}^{2 n-3}\right)$ is an epimorphism by Lemma 3.1.1). So, the direct summand $\mathbb{Z}_{4}$ of $\pi_{2 n+3}(S O(2 n-2))$ is generated by $\left[\nu_{2 n-3}^{2}\right]$. By [14], $\pi_{2 n+3}(S O(2 n+1)) \cong$ $\mathbb{Z} \oplus \mathbb{Z}_{2}$ and $\pi_{2 n+3}(S O(2 n+2)) \cong \mathbb{Z}$. It follows from $\left(\mathcal{S O}_{2 n+3}^{2 n+1}\right)$ that the direct summand $\mathbb{Z}_{2}$ of $\pi_{2 n+3}(S O(2 n+1))$ is generated by $\Delta \nu_{2 n+1}$. By [14, $\pi_{2 n+3}(S O(2 n+k-1)) \cong \mathbb{Z} \oplus \mathbb{Z}_{2}$ for $0 \leq k \leq 2$. Hence, by use of $\left(\mathcal{S O}_{2 n+3}^{2 n+k-1}\right)$ for $-1 \leq k \leq 2,\left(i_{2 n-2,2 n+1}\right)_{*}: \pi_{2 n+3}(S O(2 n-2)) \rightarrow \pi_{2 n+3}(S O(2 n+1))$ is an epimorphism and we get the relation

$$
\left(i_{2 n-2,2 n+1}\right)_{*}\left[\nu_{2 n-3}^{2}\right]=\Delta \nu_{2 n+1} .
$$

Thus, we conclude

Lemma 3.2. $\quad E^{3} J\left[\nu_{2 n-3}^{2}\right]=\left[\iota_{2 n+1}, \nu_{2 n+1}\right]$, if $n \equiv 0(\bmod 4) \geq 8$.

Next, we need

Lemma 3.3. Let $n \equiv 3(\bmod 4) \geq 7$. Then,

(1) $\left\{\Delta \iota_{n}, \eta_{n-1}, 2 \iota_{n}\right\}=0$;

(2) $\Delta\left(E\left\{\eta_{n-1}, 2 \iota_{n}, \alpha\right\}\right)=0$, where $\alpha \in \pi_{k}\left(\mathbb{S}^{n}\right)$ is an element satisfying $2 \iota_{n} \circ \alpha=0$.

Proof. By the properties of Toda brackets and the fact that $2 \pi_{n+1}(S O(n+$ $1))=0$, if $n \equiv 3(\bmod 4) \geq 7$ [14, we obtain

$i_{n+1}(\mathbb{R}) \circ\left\{\Delta \iota_{n}, \eta_{n-1}, 2 \iota_{n}\right\}=-\left\{i_{n+1}(\mathbb{R}), \Delta \iota_{n}, \eta_{n-1}\right\} \circ 2 \iota_{n+1} \subset 2 \pi_{n+1}(S O(n+1))=0$.

It follows from $\left(\mathcal{S O}_{n+1}^{n}\right)$ and (3.4) that $i_{n+1}(\mathbb{R})_{*}: \pi_{n+1}(S O(n)) \rightarrow \pi_{n+1}(S O(n+$ 1)) is a monomorphism. This leads to (1).

By (3.3) and (1), for any $\beta \in\left\{\eta_{n-1}, 2 \iota_{n}, \alpha\right\}$, we obtain

$$
\Delta(E \beta) \in \Delta \iota_{n} \circ\left\{\eta_{n-1}, 2 \iota_{n}, \alpha\right\}=-\left\{\Delta \iota_{n}, \eta_{n-1}, 2 \iota_{n}\right\} \circ E \alpha=0 .
$$

This leads to (2) and completes the proof.

We recall that $\varepsilon_{n-1} \in\left\{\eta_{n-1}, 2 \iota_{n}, \nu_{n}^{2}\right\}$ and $\mu_{n-1} \in\left\{\eta_{n-1}, 2 \iota_{n}, E^{n-5} \sigma^{\prime \prime \prime}\right\}$ for $n \geq 5$. So, by (3.1) and Lemma 3.3. (2), we obtain 
Example 3.4. Let $n \equiv 3(\bmod 4)$. Then,

(1) $\Delta \varepsilon_{n}=0$;

(2) $\Delta \mu_{n}=0$.

Hereafter, we use often the EHP sequence of the following type:

$$
\left(\mathcal{P} \mathcal{E}_{n+k}^{n}\right) \quad \pi_{n+k+2}^{2 n+1} \stackrel{P}{\longrightarrow} \pi_{n+k}^{n} \stackrel{E}{\longrightarrow} \pi_{n+k+1}^{n+1} .
$$

It is well-known that

$$
H\left[\iota_{n}, \iota_{n}\right]=0 \text { for } n \text { odd, and } H\left[\iota_{n}, \iota_{n}\right]= \pm 2 \iota_{2 n-1} \text { for } n \text { even. }
$$

So, by [31, Proposition 2.5], we obtain

Lemma 3.5 Suppose that $\pi_{n+k+2}^{2 n+1}=E^{3} \pi_{n+k-1}^{2 n-2}$ and $\pi_{n+k}^{2 n-1}=E \pi_{n+k-1}^{2 n-2}$. Then $H P\left(\pi_{n+k+2}^{2 n+1}\right)=0$ for $n$ odd and $H P\left(\pi_{n+k+2}^{2 n+1}\right)=2 \pi_{n+k}^{2 n-1}$ for $n$ even.

Suppose that $\Delta \alpha=0$ for $\alpha \in \pi_{k}\left(\mathbb{S}^{n-1}\right)$. Then, by [36], we obtain

$$
H(J[\alpha])= \pm E^{n} \alpha .
$$

Now, we show

\section{I. $\left[\iota_{n}, \nu_{n}^{2}\right] \neq 0$ if $n \equiv 1(\bmod 8) \geq 9$.}

In virtue of [31, Theorem 10.3] and its proof, $\left[\iota_{9}, \nu_{9}^{2}\right]=\bar{\nu}_{9} \nu_{17}^{2} \neq 0$.

Let $n \equiv 0(\bmod 4) \geq 8$. Assume that $E^{3}\left(J\left[\nu_{2 n-3}^{2}\right] \circ \nu_{4 n+1}\right)=0 \in$ $\pi_{4 n+7}^{2 n+1}$. Then, by use of $\left(\mathcal{P} \mathcal{E}_{4 n+6}^{2 n}\right)$ and the fact that $\left(i_{2 n-2,2 n}\right)_{*}\left[\nu_{2 n-3}^{2}\right]$ generates the direct summand $\mathbb{Z}_{2}$ of $\pi_{2 n+3}(S O(2 n))$, we obtain $E^{2}\left(J\left[\nu_{2 n-3}^{2}\right] \circ\right.$ $\left.\nu_{4 n+1}\right)=8 a\left[\iota_{2 n}, \sigma_{2 n}\right]$ for $a \in\{0,1\}$. By means of [31, Proposition 11.11.i)], there exists an element $\beta \in \pi_{4 n+4}^{2 n-2}$ such that $P\left(8 \sigma_{4 n+1}\right)=E^{2} \beta$ and $H \beta \in$ $\left\{2 \iota_{4 n-5}, \eta_{4 n-5}, 8 \sigma_{4 n-4}\right\}_{2}$. By the properties of Toda brackets, we see that

$$
\begin{array}{r}
\left\{2 \iota_{4 n-5}, \eta_{4 n-5}, 8 \sigma_{4 n-4}\right\}_{2} \subset\left\{2 \iota_{4 n-5}, \eta_{4 n-5}, 8 \sigma_{4 n-4}\right\} \subset \\
\left\{2 \iota_{4 n-5}, 0,4 \sigma_{4 n-4}\right\}=2 \iota_{4 n-5} \circ \pi_{4 n+4}^{4 n-5}+\pi_{4 n-3}^{4 n-5} \circ 4 \sigma_{4 n-3}=0 .
\end{array}
$$

So, there exists an element $\beta^{\prime} \in \pi_{4 n+3}^{2 n-3}$ such that $\beta=E \beta^{\prime}$. Hence, $E^{2}\left(J\left[\nu_{2 n-3}^{2}\right]\right.$ o $\left.\nu_{4 n+1}\right)=a E^{3} \beta^{\prime}$. 
In virtue of Lemma 1.2] (1) and (2.1), $\left[\iota_{2 n-1}, \eta_{2 n-1} \sigma_{2 n}\right]=0$. In the light of (1.2) and Example [3.4.(1), $\left[\iota_{2 n-1}, \varepsilon_{2 n-1}\right]=0$, and so $P \pi_{4 n+7}^{4 n-1}=0$. Therefore, by $\left(\mathcal{P E}_{4 n+5}^{2 n-1}\right)$,

$$
E: \pi_{4 n+5}^{2 n-1} \rightarrow \pi_{4 n+6}^{2 n} \text { is a monomorphism if } n \equiv 0(\bmod 4) \geq 8 .
$$

Hence, $E\left(J\left[\nu_{2 n-3}^{2}\right] \circ \nu_{4 n+1}\right)=a E^{2} \beta^{\prime}$. By use of $\left(\mathcal{P E}_{4 n+4}^{2 n-2}\right)$ and (3.7), we have a contradictory relation $\nu_{4 n-5}^{3}=0$. Thus, we get $\left[\iota_{2 n+1}, \nu_{2 n+1}^{2}\right]=E^{3}\left(J\left[\nu_{2 n-3}^{2}\right] \circ\right.$ $\left.\nu_{4 n+1}\right) \neq 0$.

We note the relation

$$
p_{n}(\mathbb{R})\left(\Delta \iota_{n}\right)=\left(1+(-1)^{n}\right) \iota_{n-1} .
$$

Let $n \equiv 0(\bmod 8) \geq 8$. By use of $\left(\mathcal{S O}_{n+1}^{n-1}\right)$ and [14], we get that $i_{n}(\mathbb{R})_{*}$ : $\pi_{n+1}(S O(n-1)) \rightarrow \pi_{n+1}(S O(n))$ is a monomorphism. So, we obtain

$$
\Delta \nu_{n-1}=0, \text { if } n \equiv 0(\bmod 8) \geq 8 \text {. }
$$

Hence, by Lemma 3.1 (2), $\nu_{n-1}$ and $\nu_{n-4}^{2}$ are lifted to $\left[\nu_{n-1}\right] \in \pi_{n+2}(S O(n))$ and $\left[\nu_{n-4}^{2}\right] \in \pi_{n+2}(S O(n-3))$, respectively. We show the following

Lemma 3.6. Let $n \equiv 0(\bmod 8) \geq 16$. Then, for some odd $x$,

$$
2\left[\nu_{n-1}\right]-\Delta \nu_{n}=x\left(i_{n-3, n}\right)_{*}\left[\nu_{n-4}^{2}\right] .
$$

Proof. By use of $\left(\mathcal{S O}_{n+2}^{n-k}\right)$ for $2 \leq k \leq 4$, Lemma 3.1 and [14, we see that $\left(i_{n-3, n-1}\right)_{*}: \pi_{n+2}(S O(n-3)) \rightarrow \pi_{n+2}(S O(n-1)) \cong \mathbb{Z}_{8}$ is an isomorphism and $\pi_{n+2}(S O(n-3))=\left\{\left[\nu_{n-4}^{2}\right]\right\}$. In virtue of [14], $\pi_{n+2}(S O(n+1)) \cong$ $\mathbb{Z}_{8}$ and $\pi_{n+2}(S O(n)) \cong \mathbb{Z}_{24} \oplus \mathbb{Z}_{8}$. So, by $\left(\mathcal{S O}_{n+2}^{n-k}\right)$ for $k=0,1$, we get $\pi_{n+2}(S O(n))=\left\{\Delta \nu_{n},\left[\nu_{n-1}\right]\right\}$. By (3.8), we obtain $p_{n}(\mathbb{R})\left(\Delta \nu_{n}\right)=2 \nu_{n-1}$, and hence $2\left[\nu_{n-1}\right]-\Delta \nu_{n} \in \operatorname{Im}\left(i_{n}(\mathbb{R})_{*}: \pi_{n+2}(S O(n-1)) \rightarrow \pi_{n+2}(S O(n))\right)$, where $i_{n}(\mathbb{R})_{*}$ is a split monomorphism. Since $\sharp\left(2\left[\nu_{n-1}\right]-\Delta \nu_{n}\right)=8$, we have the required relation and this completes the proof.

The relation in [31, Lemma 11.17] is regarded as the $J$-image of that in Lemma 3.6.

Now, we present a proof of the non-triviality of $\left[\iota_{n}, \nu_{n}^{2}\right]$ in the case $n \equiv$ $0(\bmod 8) \geq 8$.

II. $\left[\iota_{n}, \nu_{n}^{2}\right] \neq 0$ if $n \equiv 0(\bmod 8) \geq 8$. 
By [31, (7.19), Theorem 7.7], $\left[\iota_{8}, \nu_{8}^{2}\right]=\nu_{8} \sigma_{11} \nu_{18} \neq 0$. Let $n \equiv 0(\bmod$ $8) \geq 16$. In the light of [4, [5] and [12, $\pi_{n+5}(S O(n)) \cong\left(\mathbb{Z}_{2}\right)^{2}$. So, by (3.3) and Lemma 3.6.

$$
\Delta\left(\nu_{n}^{2}\right)=\left(i_{n-3, n}\right)_{*}\left(\left[\nu_{n-4}^{2}\right] \nu_{n+2}\right)
$$

and hence $\left[\iota_{n}, \nu_{n}^{2}\right]=E^{3}\left(J\left[\nu_{n-4}^{2}\right] \circ \nu_{2 n-1}\right)$.

Assume that $E^{3}\left(J\left[\nu_{n-4}^{2}\right] \circ \nu_{2 n-1}\right)=0$. Then, $E^{2}\left(J\left[\nu_{n-4}^{2}\right] \circ \nu_{2 n-1}\right) \in$ $P \pi_{2 n+6}^{2 n-1}=\left\{\left[\iota_{n-1}, \sigma_{n-1}\right]\right\}$. By [31, Prposition 11.11.ii)], it holds $\pi_{2 n+5}^{2 n-3} \subset$ $E^{2} \pi_{2 n+1}^{n-4}$ and in virtue of (2.4), we have $E^{2}\left(J\left[\nu_{n-4}^{2}\right] \circ \nu_{2 n-1}\right)=a E^{7}\left(\gamma \sigma_{2 n-10}\right)$ for $a \in\{0,1\}$. So, by using $\left(\mathcal{P} \mathcal{E}_{2 n+3-k}^{n-2-k}\right)$ for $k=0,1$, we get that

$$
J\left[\nu_{n-4}^{2}\right] \circ \nu_{2 n-1}-a E^{5}\left(\gamma \sigma_{2 n-10}\right)-E \beta \in P \pi_{2 n+4}^{2 n-5}
$$

for some $\beta \in \pi_{2 n+1}^{n-4}$. Hence, Lemma 3.5 and (3.7) imply a contradictory relation $\nu_{2 n-7}^{3}=0$, and thus $\left[\iota_{n}, \nu_{n}^{2}\right] \neq 0$.

We note that Nomura has a different proof from II.

\section{Proof of Theroem 2.2, part II}

Let $\omega_{n}(\mathbb{R}) \in \pi_{n-1}(O(n)), \omega_{n}(\mathbb{C}) \in \pi_{2 n}(U(n))$ and $\omega_{n}(\mathbb{H}) \in \pi_{4 n+2}(S p(n))$ be the characteristic elements for the orthogonal $O(n)$, unitary $U(n)$ and symplectic $S p(n)$ groups, respectively. We note that $\omega_{n}(\mathbb{R})=\Delta \iota_{n}$ and $\sharp\left(\Delta \iota_{n}\right)=2$ for odd $n \geq 9$.

Let $r_{n}: U(n) \rightarrow S O(2 n)$ and $c_{n}: S p(n) \rightarrow S U(2 n)$ be the canonical maps, respectively. Set $i_{n}(\mathbb{C}): U(n-1) \hookrightarrow U(n)$ for the inclusion map. As it is well-known,

$$
i_{2 n+1}(\mathbb{R}) r_{n} \omega_{n}(\mathbb{C})=\omega_{2 n+1}(\mathbb{R}) \text { and } i_{2 n+1}(\mathbb{C}) c_{n} \omega_{n}(\mathbb{H})=\omega_{2 n+1}(\mathbb{C}) .
$$

Let

$$
\tau_{2 n}^{\prime}=r_{n} \omega_{n}(\mathbb{C}) \in \pi_{2 n}(S O(2 n)) \text { and } \bar{\tau}_{4 n}^{\prime}=r_{2 n} c_{n} \omega_{n}(\mathbb{H}) \in \pi_{4 n+2}(S O(4 n)) .
$$

It is well-known that

$$
p_{2 n}(\mathbb{R}) \tau_{2 n}^{\prime}=(n-1) \eta_{2 n-1} \text { and } p_{4 n}(\mathbb{R}) \bar{\tau}_{4 n}^{\prime}= \pm(n+1) \nu_{4 n-1} \text { for } n \geq 2 \text {. }
$$

Whence, we obtain 
Lemma 4.1 (1) If $n$ is even and $n \geq 4$ then $i_{n+1}(\mathbb{R}) \tau_{n}^{\prime}=\Delta \iota_{n+1}$ and $p_{n}(\mathbb{R}) \tau_{n}^{\prime}=\left(\frac{n}{2}-1\right) \eta_{n-1}$;

(2) If $n \equiv 0(\bmod 4) \geq 8$ then $\left(i_{n, n+2}\right) \bar{\tau}_{n}^{\prime}=\tau_{n+2}^{\prime}$ and $p_{n}(\mathbb{R}) \bar{\tau}_{n}^{\prime}= \pm\left(\frac{n}{4}+\right.$ 1) $\nu_{n-1}$.

By use of $\left(\mathcal{S O}_{4 n+2}^{4 n+1}\right)$, Lemma 4.1] and [14], we obtain

$$
\Delta\left(\eta_{4 n+1}^{2}\right)=4 i_{4 n+1}(\mathbb{R}) \bar{\tau}_{4 n}^{\prime}, \text { if } n \geq 2 .
$$

So, by $\left(\mathcal{S O}_{4 n+2}^{4 n}\right)$, we have $\tau_{4 n}^{\prime} \eta_{4 n}^{2}-4 \bar{\tau}_{4 n}^{\prime}=a \Delta \nu_{4 n}$ for $a \in\{0,1, \ldots, 23\}$. Composing $p_{4 n}(\mathbb{R})$ with this relation, using the relation $\eta_{4 n-1}^{3}=12 \nu_{4 n-1}$, (3.3), (3.8) and Lemma 4.1, $a$ is even and

$$
\tau_{4 n}^{\prime} \eta_{4 n}^{2} \equiv 4 \bar{\tau}_{4 n}^{\prime}\left(\bmod 2 \Delta \nu_{4 n}\right), \text { if } n \geq 2 .
$$

Set $\tau_{2 n}=J \tau_{2 n}^{\prime} \in \pi_{4 n}\left(\mathbb{S}^{2 n}\right)$ and $\bar{\tau}_{4 n}=J \bar{\tau}_{4 n}^{\prime} \in \pi_{8 n+2}\left(\mathbb{S}^{4 n}\right)$. Then, we note that

$$
E \tau_{2 n}=\left[\iota_{2 n+1}, \iota_{2 n+1}\right], H \tau_{2 n}=(n-1) \eta_{4 n-1}
$$

and

$$
E^{3} \bar{\tau}_{4 n}=\left[\iota_{4 n+3}, \iota_{4 n+3}\right], H \bar{\tau}_{4 n}= \pm(n+1) \nu_{8 n-1}
$$

By (4.1), we have

$$
\left[\iota_{4 n+1}, \eta_{4 n+1}^{2}\right]=4 E \bar{\tau}_{4 n} .
$$

Let $\iota_{X}$ be the identity class of a space $X$. Denote by $\mathrm{P}^{n}(2)$ the Moore space of type $\left(\mathbb{Z}_{2}, n-1\right)$ and by $i_{n}: \mathbb{S}^{n-1} \hookrightarrow \mathrm{P}^{n}(2), p_{n}: \mathrm{P}^{n}(2) \rightarrow \mathbb{S}^{n}$ the inclusion and collapsing maps, respectively. We recall from [32] that

$$
2 \iota_{\mathrm{P}^{n}(2)}=i_{n} \eta_{n-1} p_{n} \text {, if } n \geq 3 .
$$

Let $\bar{\eta}_{n} \in\left[\mathrm{P}^{n+2}(2), \mathbb{S}^{n}\right] \cong \mathbb{Z}_{4}$ and $\tilde{\eta}_{n} \in \pi_{n+2}\left(\mathrm{P}^{n+1}(2)\right) \cong \mathbb{Z}_{4}$ for $n \geq 3$ be an extension and a coextension of $\eta_{n}$, respectively. We note that

$$
\bar{\eta}_{n} \in\left\{\eta_{n}, 2 \iota_{n+1}, p_{n+1}\right\} \text {, if } n \geq 3
$$

and

$$
\tilde{\eta}_{n} \in\left\{i_{n+1}, 2 \iota_{n}, \eta_{n}\right\} \text {, if } n \geq 3 .
$$


We have

$$
2 \bar{\eta}_{n}=\eta_{n}^{2} p_{n+2} \text { and } 2 \tilde{\eta}_{n}=i_{n+1} \eta_{n}^{2} \text {, if } n \geq 3
$$

We recall that $\bar{\eta}_{n} \tilde{\eta}_{n+1}= \pm E^{n-3} \nu^{\prime}$ for $n \geq 3$. Furthermore, we recall that $\pi_{n+8}\left(\mathbb{S}^{n}\right)=\left\{\varepsilon_{n}\right\} \cong \mathbb{Z}_{2}$ for $3 \leq n \leq 5$ and $\varepsilon_{3}=\left\{\eta_{3}, E \nu^{\prime}, \nu_{7}\right\}$. We need

Lemma $4.2 \varepsilon_{n}=\left\{\eta_{n} \bar{\eta}_{n+1}, \tilde{\eta}_{n+2}, \nu_{n+4}\right\}$ if $n \geq 7$.

Proof. By (4.8), we obtain

$$
\tilde{\eta}_{7} \circ \nu_{9} \in\left\{i_{8}, 2 \iota_{7}, \eta_{7}\right\} \circ \nu_{9}=-\left(i_{8} \circ\left\{2 \iota_{7}, \eta_{7}, \nu_{8}\right\}\right) \subset i_{8} \circ \pi_{12}\left(\mathbb{S}^{7}\right)=0 .
$$

So, we can take

$$
\varepsilon_{5}=\left\{\eta_{5}, 2 \nu_{6}, \nu_{9}\right\}=\left\{\eta_{5}, \bar{\eta}_{6} \tilde{\eta}_{7}, \nu_{9}\right\}=\left\{\eta_{5} \bar{\eta}_{6}, \tilde{\eta}_{7}, \nu_{9}\right\}
$$

and

$$
\varepsilon_{n} \in\left\{\eta_{n} \bar{\eta}_{n+1}, \tilde{\eta}_{n+2}, \nu_{n+4}\right\}, \text { if } n \geq 5 .
$$

The indeterminacy of this bracket is

$$
\eta_{n} \bar{\eta}_{n+1} \circ \pi_{n+8}\left(\mathrm{P}^{n+3}(2)\right)+\pi_{n+5}\left(\mathbb{S}^{n}\right) \circ \nu_{n+5} .
$$

For $n \geq 5$, by use of the homotopy exact sequence of a pair $\left(\mathrm{P}^{n+3}(2), \mathbb{S}^{n+2}\right)$, we obtain $\pi_{n+8}\left(\mathrm{P}^{n+3}(2)\right)=\left\{i_{n+3} \nu_{n+2}^{2}\right\}$, and so $\bar{\eta}_{n+1} \circ \pi_{n+8}\left(\mathrm{P}^{n+3}(2)\right)=\left\{\eta_{n+1} \nu_{n+2}^{2}\right\}=$ 0 . Hence, the indeterminacy is trivial for $n \geq 7$ and this completes the proof.

By [14], $\pi_{4 n}(S O(4 n)) \cong\left(\mathbb{Z}_{2}\right)^{3}$ or $\left(\mathbb{Z}_{2}\right)^{2}$, if $n \geq 2$. So,

$$
\sharp \tau_{4 n}^{\prime}=2 \text {, if } n \geq 2 \text {. }
$$

Now, we prove

Lemma $4.3 \tau_{n-1}^{\prime} \eta_{n-1} \varepsilon_{n} \equiv 0\left(\bmod \tau_{n-1}^{\prime} \nu_{n-1}^{3}\right)$ if $n \equiv 1(\bmod 8) \geq 17$.

Proof. By (3.3), (4.2), (4.9) and the relation $\nu_{n-1} \eta_{n+2}=0$ for $n \geq 7$, we have $\tau_{n-1}^{\prime} \eta_{n-1}^{2} \bar{\eta}_{n+1}=0$ if $n \equiv 1(\bmod 8) \geq 9$. In virtue of Lemma 4.2, we obtain

$$
\begin{array}{r}
\tau_{n-1}^{\prime} \eta_{n-1} \varepsilon_{n}=\tau_{n-1}^{\prime} \eta_{n-1} \circ\left\{\eta_{n} \bar{\eta}_{n+1}, \tilde{\eta}_{n+2}, \nu_{n+4}\right\} \\
=-\left\{\tau_{n-1}^{\prime} \eta_{n-1}, \eta_{n} \bar{\eta}_{n+1}, \tilde{\eta}_{n+2}\right\} \circ \nu_{n+5} .
\end{array}
$$


By (4.10) and noting that $2 \iota_{S O(n-1)} \circ \alpha=2 \alpha$ for any $\alpha \in \pi_{n+5}(S O(n-1))$, we obtain

$$
2\left\{\tau_{n-1}^{\prime} \eta_{n-1}, \eta_{n} \bar{\eta}_{n+1}, \tilde{\eta}_{n+2}\right\}=-\left\{2 \iota_{S O(n-1)}, \tau_{n-1}^{\prime} \eta_{n-1}, \eta_{n} \bar{\eta}_{n+1}\right\} \circ \tilde{\eta}_{n+3} .
$$

We have

$$
\left\{2 \iota_{S O(n-1)}, \tau_{n-1}^{\prime} \eta_{n-1}, \eta_{n} \bar{\eta}_{n+1}\right\} \subset\left[\mathrm{P}^{n+4}(2), S O(n-1)\right]
$$

By [14], $\pi_{n+3}(S O(n-1))=0$ if $n \equiv 1(\bmod 8) \geq 9$. So, $\left[\mathrm{P}^{n+4}(2), S O(n-\right.$ $1)]=\pi_{n+4}(S O(n-1)) \circ p_{n+4}$. In virtue of $\left(\mathcal{S O}_{n+4}^{n-k}\right)$ for $k=1,2$, [4], [5], 12] and (3.9), we see that

$$
\pi_{n+4}(S O(n-1))=\left\{\Delta\left(\nu_{n-1}\right),\left[\nu_{n-2}\right]\right\} \circ \nu_{n+1} \cong\left(\mathbb{Z}_{2}\right)^{2} .
$$

Hence, we obtain $\left[\mathrm{P}^{n+4}(2), S O(n-1)\right] \circ \tilde{\eta}_{n+3}=\pi_{n+4}(S O(n-1)) \circ \eta_{n+4}=0$. This leads to the relation

$$
2\left\{\tau_{n-1}^{\prime} \eta_{n-1}, \eta_{n} \bar{\eta}_{n+1}, \tilde{\eta}_{n+2}\right\}=0 .
$$

By [4, [5] and [12, we see that

$$
\pi_{n+5}(S O(n)) \cong \mathbb{Z}_{16} \oplus \mathbb{Z}_{2}, \text { if } n \equiv 1(\bmod 8) \geq 17
$$

where the direct summand $\mathbb{Z}_{2}$ is generated by $\Delta\left(\nu_{n}^{2}\right)$ and

$$
\pi_{n+5}(S O(n-1)) \cong \begin{cases}\left(\mathbb{Z}_{16}\right)^{2} \oplus \mathbb{Z}_{2} \oplus \mathbb{Z}_{15}, & \text { if } n \equiv 1(\bmod 16) \geq 17 \\ \mathbb{Z}_{32} \oplus \mathbb{Z}_{8} \oplus \mathbb{Z}_{2} \oplus \mathbb{Z}_{15}, & \text { if } n \equiv 9(\bmod 16) \geq 25\end{cases}
$$

where the direct summand $\mathbb{Z}_{2}$ is generated by $\tau_{n-1}^{\prime} \nu_{n-1}^{2}$. Thus, for $n \equiv$ $1(\bmod 16) \geq 17$, we obtain

$$
\left\{\tau_{n-1}^{\prime} \eta_{n-1}, \eta_{n} \bar{\eta}_{n+1}, \tilde{\eta}_{n+2}\right\} \subset\left\{\tau_{n-1}^{\prime} \nu_{n-1}^{2}\right\}+8 \pi_{n+5}(S O(n-1)),
$$

and so $\tau_{n-1}^{\prime} \eta_{n-1} \varepsilon_{n} \equiv 0\left(\bmod \tau_{n-1}^{\prime} \nu_{n-1}^{3}\right)$. For $n \equiv 9(\bmod 16) \geq 25$, by $\left(\mathcal{S O}_{n+5}^{n-1}\right)$, we get

$$
\pi_{n+5}(S O(n-1) ; 2)=\left\{\beta, \Delta \sigma_{n-1}-2 \beta, \tau_{n-1}^{\prime} \nu_{n-1}^{2}\right\} \cong \mathbb{Z}_{32} \oplus \mathbb{Z}_{8} \oplus \mathbb{Z}_{2},
$$

where $\beta$ is such an element that $16 \beta=8 \Delta \sigma_{n-1}$ and $i_{n}(\mathbb{R}) \beta$ is a generator of the direct summand $\mathbb{Z}_{16}$ of $\pi_{n+5}(S O(n))$. Then, by (3.3) and the relation $\sigma_{n-1} \nu_{n+6}=0$ for $n \geq 13$ [31, (7.20)], we have

$$
4\left(\Delta \sigma_{n-1}-2 \beta\right) \circ \nu_{n+5}=4 \Delta\left(\sigma_{n-1} \nu_{n+6}\right)+\beta \circ 8 \nu_{n+5}=0 .
$$


Consequently, we obtain the relation $\tau_{n-1}^{\prime} \eta_{n-1} \varepsilon_{n} \equiv 0\left(\bmod \tau_{n-1}^{\prime} \nu_{n-1}^{3}\right)$ if $n \equiv$ $9(\bmod 16) \geq 25$ and this completes the proof.

Next, we show

Lemma 4.4. If $n \equiv 0,1(\bmod 4) \geq 8$ then $\left[\iota_{n}, \alpha\right] \neq 0$ for $\alpha=\varepsilon_{n}, \bar{\nu}_{n}$ and $\eta_{n} \sigma_{n+1}$.

Proof. We show $\left[\iota_{n}, \varepsilon_{n}\right] \neq 0$. Let $n \equiv 0(\bmod 4) \geq 8$. By 31, Proposition 11.10.i)], there exists an element $\beta \in \pi_{2 n+6}^{n-1}$ such that $E \beta=\left[\iota_{n}, \varepsilon_{n}\right]$ and $H \beta=\eta_{2 n-3} \varepsilon_{2 n-2}$. Assume that $\left[\iota_{n}, \varepsilon_{n}\right]=0$. Then, by $\left(\mathcal{P} \mathcal{E}_{2 n+6}^{n-1}\right)$, we have $\beta \in P \pi_{2 n+8}^{2 n-1}$. This induces a contradictory relation $\eta_{2 n-3} \varepsilon_{2 n-2}=0$, and hence $\left[\iota_{n}, \varepsilon_{n}\right] \neq 0$. Next, consider the case $n \equiv 1(\bmod 4) \geq 9$. Then, by (4.3),$\left[\iota_{n}, \varepsilon_{n}\right]=E\left(\tau_{n-1} \varepsilon_{2 n-2}\right)$ and $H\left(\tau_{n-1} \varepsilon_{2 n-2}\right)=\eta_{2 n-3} \varepsilon_{2 n-2}$. Assume that $\left[\iota_{n}, \varepsilon_{n}\right]=0$. Then, $\left(\mathcal{P} \mathcal{E}_{2 n+6}^{n-1}\right)$ and Lemma 3.5 lead to a contradictory relation $\eta_{2 n-3} \varepsilon_{2 n-2}=0$, and so $\left[\iota_{n}, \varepsilon_{n}\right] \neq 0$. For other elements, the argument goes ahead similarly.

By (1.3) and Lemma 4.4, $\Delta: \pi_{n+8}\left(\mathbb{S}^{n}\right) \rightarrow \pi_{n+7}(S O(n))$ is a monomorphism, if $n \equiv 0,1(\bmod 4) \geq 12$. So, by $\left(\mathcal{S O}_{n+8}^{n}\right)$, we obtain the exact sequence

$$
\begin{gathered}
\pi_{n+9}\left(\mathbb{S}^{n}\right) \stackrel{\Delta}{\longrightarrow} \pi_{n+8}(S O(n)) \stackrel{i_{*}}{\longrightarrow} \pi_{n+8}(S O(n+1)) \longrightarrow 0 \\
\text { if } n \equiv 0,1(\bmod 4) \geq 12 .
\end{gathered}
$$

We recall from [19] and [31] the following:

$$
\begin{gathered}
\sharp\left[\iota_{n}, \mu_{n}\right]= \begin{cases}1, & \text { if } n=2,6 \text { or } n \equiv 3(\bmod 4) ; \\
2, & \text { if } n \equiv 0,1,2(\bmod 4) \geq 4 \text { unless } n=6,\end{cases} \\
\sharp\left[\iota_{n}, \eta_{n} \mu_{n+1}\right]= \begin{cases}1, & \text { if } n=5 \text { or } n \equiv 2,3(\bmod 4) ; \\
2, & \text { if } n \equiv 0,1(\bmod 4) \geq 4 \text { unless } n=5,\end{cases} \\
\sharp\left[\iota_{n}, \eta_{n}^{2} \sigma_{n+2}\right]= \begin{cases}1, & \text { if } n \equiv 2,3(\bmod 4) \geq 6 ; \\
2, & \text { if } n \equiv 0(\bmod 4) \geq 8\end{cases}
\end{gathered}
$$

and

$$
(*) \quad \sharp\left[\iota_{n}, \eta_{n}^{2} \sigma_{n+2}\right]=2 \text {, if } n \equiv 1(\bmod 8) \geq 17 .
$$

Now, we conclude 
Corollary 4.5. (1) $\left[\iota_{n}, \eta_{n} \varepsilon_{n+1}\right] \equiv 0\left(\bmod \left[\iota_{n}, \nu_{n}^{3}\right]\right)$ if $n \equiv 1(\bmod 8) \geq 9$;

(2) $\left[\iota_{n}, \nu_{n}^{3}\right]=0$ if $n \equiv 5(\bmod 8)$ and $\left[\iota_{n}, \eta_{n} \varepsilon_{n+1}\right]=\left[\iota_{n}, \eta_{n}^{2} \sigma_{n+2}\right]=0$ provided $n \equiv 5(\bmod 8) \geq 13$ unless $n \equiv 53(\bmod 64)$.

Proof. We have $\left[\iota_{9}, \eta_{9} \varepsilon_{10}\right]=\eta_{9} \sigma_{10} \eta_{17} \varepsilon_{18}+\sigma_{9} \eta_{16}^{2} \varepsilon_{18}=\eta_{9}^{2} \sigma_{11} \varepsilon_{18}+4 \sigma_{9} \nu_{16} \sigma_{19}$ $=0$. For the case $n \equiv 1(\bmod 8) \geq 17,(1)$ is a direct consequence of Lemma 4.3.

By (3.3) and Lemma 3.1.(1), we have $\Delta\left(\nu_{n}^{3}\right)=0$. So, the first assertion of (2) holds. In the light of [22], the second assertion of (2) holds for $n=13$. Let $n \equiv 5(\bmod 8) \geq 21$. We consider the exact sequence (4.12). By [4], [5] and [12], we see that

$$
\pi_{n+8}(S O(n+1)) \cong \begin{cases}\mathbb{Z}_{4} \oplus \mathbb{Z}_{2}, & \text { if } n \equiv 5(\bmod 32) \geq 37 \\ \left(\mathbb{Z}_{4}\right)^{2}, & \text { if } n \equiv 21(\bmod 32) \\ \mathbb{Z}_{4}, & \text { if } n \equiv 13(\bmod 16)\end{cases}
$$

and

$$
\pi_{n+8}(S O(n)) \cong \begin{cases}\mathbb{Z}_{4} \oplus\left(\mathbb{Z}_{2}\right)^{2}, & \text { if } n \equiv 5(\bmod 32) \geq 37 \\ \left(\mathbb{Z}_{4}\right)^{2} \oplus \mathbb{Z}_{2}, & \text { if } n \equiv 21(\bmod 64) ; \\ \mathbb{Z}_{8} \oplus \mathbb{Z}_{4} \oplus \mathbb{Z}_{2}, & \text { if } n \equiv 53(\bmod 64) ; \\ \mathbb{Z}_{4} \oplus \mathbb{Z}_{2}, & \text { if } n \equiv 13(\bmod 16) .\end{cases}
$$

By (4.13) and (4.14), $\left[\iota_{n}, \mu_{n}\right] \neq 0$ and $\left[\iota_{n}, \mu_{n}\right] \eta_{2 n+8} \neq 0$. So, by the group structures of $\pi_{n+8}(S O(n+k))$ for $k=0,1$, we get that $\Delta \mu_{n}$ is taken as a generator of the direct summand $\mathbb{Z}_{2}$ of $\pi_{n+8}(S O(n))$. By (3.3) and (4.1), we obtain

$$
\Delta\left(\eta_{n}^{2} \sigma_{n+2}\right)=4 i_{n}(\mathbb{R}) \bar{\tau}_{n-1}^{\prime} \sigma_{n+1}
$$

and hence

$$
\Delta\left(\eta_{n}^{2} \sigma_{n+2}\right)= \begin{cases}0, & \text { if } n \not \equiv 53(\bmod 64) \\ 4 i_{n}(\mathbb{R}) \bar{\tau}_{n-1}^{\prime} \sigma_{n+1} \neq 0, & \text { if } n \equiv 53(\bmod 64)\end{cases}
$$

This leads to the second assertion of (2) and the proof is complete.

By [27, Proposition 4.2],

$$
\left[\iota_{n}, \eta_{n} \varepsilon_{n+1}\right] \equiv 0\left(\bmod \left[\iota_{n}, \eta_{n}^{2} \sigma_{n+2}\right]\right), \text { if } n \equiv 1(\bmod 8) \geq 9 .
$$

Thus,

$$
\left[\iota_{n}, \eta_{n} \varepsilon_{n+1}\right]=0, \quad \text { if } \quad n \equiv 1(\bmod 8) \geq 9 .
$$

Next, we prove 
Lemma 4.6. Let $n \equiv 1(\bmod 4) \geq 5$. Then $E\left(\bar{\tau}_{2 n-2} \nu_{4 n-2}^{2}\right)=\left[\iota_{2 n-1}, \bar{\nu}_{2 n-1}\right]$ if and only if $\left[\iota_{2 n+1}, \nu_{2 n+1}^{2}\right]=0$.

Proof. By (4.4), $E^{3}\left(\bar{\tau}_{2 n-2} \nu_{4 n-2}^{2}\right)=\left[\iota_{2 n+1}, \nu_{2 n+1}^{2}\right]$. This induces the necessary condition.

Now, suppose that $\left[\iota_{2 n+1}, \nu_{2 n+1}^{2}\right]=0$. Then, by $\left(\mathcal{P} \mathcal{E}_{4 n+6}^{2 n}\right), E^{2}\left(\bar{\tau}_{2 n-2} \nu_{4 n-2}^{2}\right) \in$ $P \pi_{4 n+8}^{4 n+1} \cong \mathbb{Z}_{16}$. We assume that $E^{2}\left(\bar{\tau}_{2 n-2} \nu_{4 n-2}^{2}\right)=8 a P\left(\sigma_{4 n+1}\right)$ for $a \in\{0,1\}$. By [31, Proposition 11.11.ii)], there exists an element $\beta \in \pi_{4 n+4}^{2 n-2}$ such that

$$
P\left(8 \sigma_{4 n+1}\right)=E^{2} \beta \quad \text { and } \quad H \beta \in\left\{\eta_{4 n-5}, 2 \iota_{4 n-4}, 8 \sigma_{4 n-4}\right\}_{2} .
$$

We recall that

$$
\mu_{4 n-5} \in\left\{\eta_{4 n-5}, 2 \iota_{4 n-4}, 8 \sigma_{4 n-4}\right\}_{2}\left(\bmod \nu_{4 n-5}^{3}, \eta_{4 n-5} \varepsilon_{4 n-4}\right) .
$$

Thus, we get

$$
H \beta=\mu_{4 n-5}+x \nu_{4 n-5}^{3}+y \eta_{4 n-5} \varepsilon_{4 n-4}(x, y \in\{0,1\}) .
$$

By using $\left(\mathcal{P} \mathcal{E}_{4 n+5}^{2 n-1}\right)$ and the assumption, we have

$$
E\left(\bar{\tau}_{2 n-2} \nu_{4 n-2}^{2}\right)-a E \beta \in P \pi_{4 n+7}^{4 n-1}=\left\{P\left(\bar{\nu}_{4 n-1}\right), P\left(\varepsilon_{4 n-1}\right)\right\} .
$$

By (4.3),$P\left(\bar{\nu}_{4 n-1}\right)=E\left(\tau_{2 n-2} \bar{\nu}_{4 n-4}\right)$ and $P\left(\varepsilon_{4 n-1}\right)=E\left(\tau_{2 n-2} \varepsilon_{4 n-4}\right)$. So, by $\left(\mathcal{P E}_{4 n+4}^{2 n-2}\right)$,

$$
\bar{\tau}_{2 n-2} \nu_{4 n-2}^{2}-a \beta-b \tau_{2 n-2} \bar{\nu}_{4 n-4}-c \tau_{2 n-2} \varepsilon_{4 n-4} \in P \pi_{4 n+6}^{4 n-3}(b, c \in\{0,1\}) .
$$

Applying $H: \pi_{4 n+4}^{2 n-2} \rightarrow \pi_{4 n+4}^{4 n-5}$ to this equation, using (4.3), Lemma 3.5 and the relation $\eta_{4 n-5} \bar{\nu}_{4 n-4}=\nu_{4 n-5}^{3}$, we obtain

$$
\nu_{4 n-5}^{3}+a\left(\mu_{4 n-5}+x \nu^{3} 4 n-5+y \eta_{4 n-5} \varepsilon_{4 n-4}\right)+b \nu_{4 n-5}^{3}+c \eta_{4 n-5} \varepsilon_{4 n-4}=0 .
$$

By the group structure of $\pi_{4 n+4}^{4 n-5}, a=c=0$ and $b=1$, and so $E\left(\bar{\tau}_{2 n-2} \nu_{4 n-2}^{2}\right)=$ $E\left(\tau_{2 n-2} \bar{\nu}_{4 n-4}\right)$. Whence the proof is complete.

Since $\nu_{n} \eta_{n+3}=0$ and $\bar{\nu}_{n} \eta_{n+8}=\nu_{n}^{3}$ for $n \geq 6$, Lemma 4.6 implies

Corollary 4.7. If $\left[\iota_{8 n+3}, \nu_{8 n+3}^{2}\right]=0$, then $\left[\iota_{8 n+1}, \nu_{8 n+1}^{3}\right]=0$. 
Now, we show

III. $\left[\iota_{n}, \nu_{n}^{2}\right]=0$ if $n=2^{i}-5(i \geq 4)$.

We recall the Mahowald element $\eta_{i}^{\prime} \in \pi_{2^{i}}^{S}\left(\mathbb{S}^{0}\right)$ for $i \geq 3[20$. We set $\eta_{i-1, m}^{\prime}=\eta_{i-1}^{\prime}$ on $\mathbb{S}^{m}$ for $m=2^{i-1}-2$ with $i \geq 4$, that is, $\eta_{i-1, m}^{\prime} \in \pi_{2^{i-1}+m}\left(\mathbb{S}^{m}\right)$. It satisfies the relation $H\left(\eta_{i-1, m}^{\prime}\right)=\nu_{2 m-1}$. Then, the assertion follows directly from [3].

Finally, we show

\section{IV. $\left[\iota_{n}, \nu_{n}^{2}\right] \neq 0$ if $n \equiv 3(\bmod 8) \geq 19$ unless $n=2^{i}-5$.}

By III and Corollary 4.7, we obtain

$$
\left[\iota_{n}, \nu_{n}^{3}\right]=0, \quad \text { if } \quad n=2^{i}-7(i \geq 4) .
$$

Hence, from (4.17) and the relation $\eta_{n}^{2} \sigma_{n+2}=\nu_{n}^{3}+\eta_{n} \varepsilon_{n+1}$,

$$
\left[\iota_{n}, \eta_{n}^{2} \sigma_{n+2}\right]=0 \text {, if } n=2^{i}-7(i \geq 4) .
$$

Let $n \equiv 1(\bmod 8) \geq 17$. Considering the exact sequence (4.12), in virtue of [4, 5] and [12, we obtain

$$
\pi_{n+8}(S O(n)) \cong \mathbb{Z}_{2} \oplus \mathbb{Z}_{2} \oplus \mathbb{Z}_{8} \quad \text { and } \quad \pi_{n+8}(S O(n+1)) \cong \mathbb{Z}_{2} \oplus \mathbb{Z}_{4} .
$$

By (4.13), (4.14) and $(*)$, we know that

$$
\left[\iota_{n}, \mu_{n}\right] \neq 0,\left[\iota_{n}, \mu_{n}\right] \eta_{2 n+8} \neq 0
$$

and

$$
\left[\iota_{n}, \eta_{n}^{2} \sigma_{n+2}\right] \neq 0
$$

So, by (4.5), we get the relation

$$
4 E\left(\bar{\tau}_{n-1} \sigma_{2 n}\right)=\left[\iota_{n}, \eta_{n}^{2} \sigma_{n+2}\right] \neq 0 .
$$

By $(*)$ and (4.17), we obtain

$$
\left[\iota_{n}, \nu_{n}^{3}\right]=\left[\iota_{n}, \eta_{n}^{2} \sigma_{n+2}\right] \neq 0, \text { if } n \equiv 1(\bmod 8) \geq 17 \text { and } n \neq 2^{i}-7 .
$$

Thus, by Corollary 4.7, we obtain the assertion.

We are in a position to assert that Mahowald's result [19] should be stated as follows.

Theorem 4.8. Let $n \equiv 1(\bmod 8) \geq 9$. Then $\left[\iota_{n}, \eta_{n}^{2} \sigma_{n+2}\right] \neq 0$ if and only if $n \neq 2^{i}-7$. 


\section{Proof of $\left[\iota_{16 s+7}, \sigma_{16 s+7}\right] \neq 0$ for $s \geq 1$}

We give a proof of the first part of Theorem 2.4. First of all, let $n \equiv$ $2(\bmod 4) \geq 10$. Then, by use of $\left(\mathcal{S O}_{n}^{n}\right)$, Lemma 4.1. (1) and [14], we obtain $\pi_{n}(S O(n))=\left\{\tau_{n}^{\prime}\right\} \cong \mathbb{Z}_{4}$ and

$$
2 \tau_{n}^{\prime}=\Delta \eta_{n}, \text { if } n \equiv 2(\bmod 4) \geq 10 .
$$

We recall from [31, p.95-6] the construction of the element $\kappa_{7} \in \pi_{21}\left(\mathbb{S}^{7}\right)$. It is a representative of a Toda bracket

$$
\left\{\nu_{7}, E \alpha, E^{2} \beta\right\}_{1},
$$

where $\alpha=\bar{\eta}_{9} \in\left[\mathrm{P}^{11}(2), \mathbb{S}^{9}\right]$ is an extension of $\eta_{9}$ and $\beta=\widetilde{\bar{\nu}}_{9} \in \pi_{18}\left(\mathrm{P}^{10}(2)\right)$ is a coextension of $\bar{\nu}_{9}$ satisfying $\alpha \circ E \beta=0$. Furthermore, $\kappa_{n}=E^{n-7} \kappa_{7}$ for $n \geq 7$ and set $\widetilde{\bar{\nu}}_{n}=E^{n-9} \widetilde{\bar{\nu}}_{9}$ for $n \geq 9$. Then, we can take

$$
\kappa_{n} \in\left\{\nu_{n}, \bar{\eta}_{n+3}, \widetilde{\bar{\nu}}_{n+4}\right\} \text { for } n \geq 7 \text {. }
$$

By [14, $\pi_{n+4}(S O(n+k)) \cong \mathbb{Z} \oplus \mathbb{Z}_{2}$ for $k=1,2$ if $n \equiv 7(\bmod 8)$. And, by $\left(\mathcal{S O}_{n+4}^{n+2}\right)$, the direct summand $\mathbb{Z}_{2}$ of $\pi_{n+4}(S O(n+2))$ is generated by $\Delta \nu_{n+2}$. So, the non-triviality of $\left[\nu_{n}\right] \eta_{n+3} \in \pi_{n+4}(S O(n+1))$ induces the relation $i_{n+2}(\mathbb{R})_{*}\left(\left[\nu_{n}\right] \eta_{n+3}\right)=\Delta \nu_{n+2}$. Because of the fact that $\left[\iota_{n+2}, \nu_{n+2}^{2}\right] \neq 0$, this induces a contradictory relation $0=\Delta \nu_{n+2}^{2} \neq 0$. Hence, we obtain

$$
\left[\nu_{n}\right] \eta_{n+3}=0, \text { if } n \equiv 7(\bmod 8) \text {. }
$$

Next, by [14,

$$
\left\{\left[\nu_{n}\right], \eta_{n+3}, 2 \iota_{n+4}\right\} \subset \pi_{n+5}(S O(n+1))=0 \text {, if } n \equiv 7(\bmod 8) .
$$

So, by (4.7), we have $\left[\nu_{n}\right] \bar{\eta}_{n+3} \in\left\{\left[\nu_{n}\right], \eta_{n+3}, 2 \iota_{n+4}\right\} \circ p_{n+5}=0$ and hence we can define a lift of $\kappa_{n}$ for $n \equiv 7(\bmod 8)$, as follows:

$$
\left[\kappa_{n}\right] \in\left\{\left[\nu_{n}\right], \bar{\eta}_{n+3}, \widetilde{\bar{\nu}}_{n+4}\right\} \subset \pi_{n+14}(S O(n+1)) \text { for } n \equiv 7(\bmod 8) .
$$

Let $n \equiv 7(\bmod 8) \geq 15$. By use of $\left(\mathcal{S O}_{n-4}^{n-4}\right),\left(\mathcal{S O}_{n-3}^{n-k}\right)$ for $k=2,3,5$, $\left(\mathcal{S O}_{n-2}^{n-l}\right)$ for $2 \leq l \leq 5$ and [14, we obtain

$$
\pi_{n-4}(S O(n-4))=\{\beta\} \cong \mathbb{Z} ; \pi_{n-3}(S O(n-4))=\left\{\left[\eta_{n-5}^{2}\right]\right\} \cong \mathbb{Z}_{2} ;
$$




$$
\begin{gathered}
\pi_{n-2}(S O(n-4))=\left\{\left[\eta_{n-5}^{2}\right] \eta_{n-3}, \Delta \nu_{n-4}\right\} \cong\left(\mathbb{Z}_{2}\right)^{2} ; \\
\pi_{n-4}(S O(n-3))=\left\{i_{n-3}(\mathbb{R}) \beta, \Delta \iota_{n-3}\right\} \cong(\mathbb{Z})^{2} ; \\
\pi_{n-3}(S O(n-3))=\left\{\left[\eta_{n-4}\right], \Delta \eta_{n-3}\right\} \cong\left(\mathbb{Z}_{2}\right)^{2} ; \\
\pi_{n-2}(S O(n-3))=\left\{\left[\eta_{n-4}\right] \eta_{n-4}, \Delta \eta_{n-3}^{2}\right\} \cong\left(\mathbb{Z}_{2}\right)^{2} ; \\
\pi_{n-2}(S O(n-2))=\left\{\Delta \eta_{n-2}\right\} \cong\left(\mathbb{Z}_{2}\right)^{2},
\end{gathered}
$$

where $\beta$ is a generator of $\pi_{n-4}(S O(n-4))$ and

$$
\Delta \eta_{n-3}=i_{n-3}(\mathbb{R})\left[\eta_{n-5}^{2}\right] .
$$

We need

$$
\left\{p_{n}(\mathbb{R}), i_{n}(\mathbb{R}), \Delta \iota_{n-1}\right\} \ni \iota_{n-1}\left(\bmod 2 \iota_{n-1}\right) \text { for } n \geq 9 .
$$

Since $\sharp\left[\eta_{n-4}\right]=2$ for $n \equiv 7(\bmod 8),\left[\eta_{n-4}\right]$ is lifted to $\overline{\left[\eta_{n-4}\right]} \in\left[\mathrm{P}^{n-2}(2), S O(n-\right.$ $4)$. Since $p_{n-3}(\mathbb{R}) \beta=0$, we obtain

$$
\beta \eta_{n-4}=0 \in \pi_{n-3}(S O(n-4)) .
$$

So, by (5.2) and (5.3), we get

$$
\begin{aligned}
& {\left[\eta_{n-4}\right] \in\left\{i_{n-3}(\mathbb{R}), \Delta \iota_{n-4}, \eta_{n-5}\right\}\left(\bmod i_{n-3}(\mathbb{R}) \circ \pi_{n-3}(S O(n-4))\right.} \\
& \left.+\pi_{n-4}(S O(n-3)) \circ \eta_{n-4}=\left\{\Delta \eta_{n-3}\right\}\right) \text { for } n \equiv 7(\bmod 8) \geq 15
\end{aligned}
$$

By the same reason as (3.1), we obtain $\Delta\left(\bar{\eta}_{3}\right)=0 \in\left[\mathrm{P}^{4}(2), S O(3)\right]$. Let $n \equiv 7(\bmod 8) \geq 15$. Then, by Lemma 3.3. (1) and (4.7), we obtain

$$
\Delta\left(\bar{\eta}_{n-4}\right)=\Delta \iota_{n-4} \circ \bar{\eta}_{n-5} \in-\left\{\Delta \iota_{n-4}, \eta_{n-5}, 2 \iota_{n-4}\right\} \circ p_{n-3}=0 .
$$

So, $\bar{\eta}_{n-4}$ is lifted to $\left[\bar{\eta}_{n-4}\right] \in\left[\mathrm{P}^{n-2}(2), S O(n-3)\right]$ for $n \equiv 7(\bmod 8)$. We note $\left[\bar{\eta}_{n-4}\right] \in\left\{i_{n-3}(\mathbb{R}), \Delta \iota_{n-4}, \bar{\eta}_{n-5}\right\}$ for $n \equiv 7(\bmod 8) \geq 15$. We show

Lemma 5.1. $\quad$ Let $n \equiv 7(\bmod 8) \geq 15$.

(1) $\overline{\left[\eta_{n-4}\right]} \in\left\{i_{n-3}(\mathbb{R}), \Delta \iota_{n-4}, \bar{\eta}_{n-5}\right\}\left(\bmod \left\{\Delta \bar{\eta}_{n-3}\right\}+\pi_{n-2}(S O(n-3)) \circ\right.$ $\left.p_{n-2}+K\right)$, where $K=i_{n-3}(\mathbb{R})_{*}\left[\mathrm{P}^{n-2}(2), S O(n-4)\right]+\pi_{n-4}(S O(n-3)) \circ \bar{\eta}_{n-4}$.

(2) $i_{n-2}(\mathbb{R})_{*} K \subset\left\{\left(\Delta \eta_{n-2}\right) p_{n-2}\right\}$. 
Proof. By use of the cofiber sequence $\mathbb{S}^{n-3} \stackrel{i_{n-2}}{\longrightarrow} \mathrm{P}^{n-2}(2) \stackrel{p_{n-2}}{\longrightarrow} \mathbb{S}^{n-2}$, (4.6) and (5.5), we get that $\overline{\left[\eta_{n-4}\right]} \in\left\{i_{n-3}(\mathbb{R}), \Delta \iota_{n-4}, \bar{\eta}_{n-5}\right\}\left(\bmod \left\{\Delta \bar{\eta}_{n-3}\right\}+\right.$ $\left.\pi_{n-2}(S O(n-3)) \circ p_{n-2}+K\right)$ and that $\left[\mathrm{P}^{n-2}(2), S O(n-4)\right]=\left\{\overline{\left[\eta_{n-5}^{2}\right]}\right.$, $\left.\left(\Delta \nu_{n-4}\right) p_{n-2}\right\} \cong \mathbb{Z}_{4} \oplus \mathbb{Z}_{2}$, where $\overline{\left[\eta_{n-5}^{2}\right]}$ is an extension of $\left[\eta_{n-5}^{2}\right]$ and $\overline{2\left[\eta_{n-5}^{2}\right]}=$ $\left[\eta_{n-5}^{2}\right] \eta_{n-3} p_{n-2}$. Hence, by (5.2), we see that

$$
\begin{gathered}
i_{n-4, n-2_{*}} \overline{\left[\eta_{n-5}^{2}\right]} \in i_{n-2}(\mathbb{R}) \circ\left\{\Delta \eta_{n-3}, 2 \iota_{n-3}, p_{n-3}\right\}= \\
-\left\{i_{n-2}(\mathbb{R}), \Delta \eta_{n-3}, 2 \iota_{n-3}\right\} \circ p_{n-2} .
\end{gathered}
$$

Since $\left\{i_{n-2}(\mathbb{R}), \Delta \eta_{n-3}, 2 \iota_{n-3}\right\} \subset \pi_{n-2}(S O(n-2))=\left\{\Delta \eta_{n-2}\right\}$, we have $i_{n-4, n-2 *}\left[\mathrm{P}^{n-2}(2), S O(n-\right.$ 4) $] \subset\left\{\left(\Delta \eta_{n-2}\right) p_{n-2}\right\}$.

By (4.7) and (15.4), we have $\beta \bar{\eta}_{n-4} \in\left\{\beta, \eta_{n-4}, 2 \iota_{n-3}\right\} \circ p_{n-2} \subset \pi_{n-2}(S O(n-$ $2)) \circ p_{n-2}$. Hence, we obtain $i_{n-2}(\mathbb{R})_{*}\left(\pi_{n-4}(S O(n-3)) \circ \bar{\eta}_{n-4}\right) \subset\left\{\left(\Delta \eta_{n-2}\right) p_{n-2}\right\}$. This completes the proof.

We show

Lemma 5.2. $\quad\left(i_{n-7, n-1}\right)_{*}\left[\kappa_{n-8}\right]=\Delta \bar{\nu}_{n-1}$ if $n \equiv 7(\bmod 8) \geq 15$.

Proof. By the group structures of $\pi_{n-5}(S O(n-7+k))$ for $0 \leq k \leq 3$ [14], we have $\left(i_{n-7, n-4}\right)_{*}\left[\nu_{n-8}\right]=\Delta \iota_{n-4}$, and so

$$
\left(i_{n-7, n-1}\right)_{*}\left[\kappa_{n-8}\right] \in\left(i_{n-4, n-1}\right)_{*}\left\{\Delta \iota_{n-4}, \bar{\eta}_{n-5}, \widetilde{\bar{\nu}}_{n-4}\right\} .
$$

By Lemma 5.1, we obtain

$$
\begin{array}{r}
i_{n-3}(\mathbb{R})_{*}\left\{\Delta \iota_{n-4}, \bar{\eta}_{n-5}, \widetilde{\bar{\nu}}_{n-4}\right\}=-\left\{i_{n-3}(\mathbb{R}), \Delta \iota_{n-4}, \bar{\eta}_{n-5}\right\} \circ \widetilde{\bar{\nu}}_{n-3} \equiv \\
\overline{\left[\eta_{n-4}\right]} \circ \widetilde{\bar{\nu}}_{n-3} \in\left\{\left[\eta_{n-4}\right], 2 \iota_{n-3}, \bar{\nu}_{n-3}\right\}\left(\bmod \left[\eta_{n-4}\right] \circ \pi_{n+6}\left(\mathbb{S}^{n-3}\right)\right. \\
\left.+\pi_{n-2}(S O(n-3)) \circ \bar{\nu}_{n-2}+K \circ \widetilde{\widetilde{\nu}}_{n-3}\right) .
\end{array}
$$

From the relation $i_{n-2}(\mathbb{R})\left[\eta_{n-4}\right]=\Delta \iota_{n-2}$, we see that

$$
\begin{aligned}
\left(i_{n-7, n-1}\right)_{*}\left[\kappa_{n-8}\right] & \in-i_{n-1}(\mathbb{R}) \circ\left\{\Delta \iota_{n-2}, 2 \iota_{n-3}, \bar{\nu}_{n-3}\right\} \\
& =\left\{i_{n-1}(\mathbb{R}), \Delta \iota_{n-2}, 2 \iota_{n-3}\right\} \circ \bar{\nu}_{n-2} .
\end{aligned}
$$

We note $i_{n-2}(\mathbb{R})_{*}\left(K \circ \widetilde{\bar{\nu}}_{n-3}\right) \subset\left\{\Delta \eta_{n-2}\right\} \circ \bar{\nu}_{n-3}=\left\{\Delta \nu_{n-2}^{3}\right\}=0$ by Lemma 5.1 and (3.5). Since $\left\{i_{n-1}(\mathbb{R}), \Delta \iota_{n-2}, 2 \iota_{n-3}\right\} \equiv \Delta \iota_{n-1}\left(\bmod 2 \Delta \iota_{n-1}\right)$ by (15.3), we have

$$
\left\{i_{n-1}(\mathbb{R}), \Delta \iota_{n-2}, 2 \iota_{n-3}\right\} \circ \bar{\nu}_{n-2}=\Delta \bar{\nu}_{n-1} .
$$


This completes the proof.

We can take $\left[\bar{\nu}_{n}\right] \in\left\{\left[\nu_{n}\right], \eta_{n+3}, \nu_{n+4}\right\}$ for $n \equiv 7(\bmod 8)$. And we obtain $2\left[\bar{\nu}_{n}\right]=0$ and $2\left[\kappa_{n}\right] \equiv\left[\bar{\nu}_{n}\right] \nu_{n+8}^{2}\left(\bmod \left[\nu_{n}\right] \zeta_{n+3}\right)$. By using these facts and the group structures of $\pi_{n+k}(S O(n+1))$ for $k=11,12$ and $n \equiv 7(\bmod 8) \geq 15$, we obtain

Remark 5.3 A lift $\left[\kappa_{n}\right] \in \pi_{n+14}(S O(n+1))$ of $\kappa_{n}$ is taken so that its order is two for $n \equiv 7(\bmod 8) \geq 15$.

Let $n \equiv 2(\bmod 4) \geq 6$. By the relation $4 \zeta_{n}=\eta_{n}^{2} \mu_{n+2}$, Lemma 1.2. (1) and (2.2), $4\left[\iota_{n}, \zeta_{n}\right]=0$. So, by the relation $H\left[\iota_{n}, \zeta_{n}\right]= \pm 2 \zeta_{2 n-1}$, we obtain

$$
\sharp\left[\iota_{n}, \zeta_{n}\right]=4 \text {, if } n \equiv 2(\bmod 4) \geq 6 .
$$

By [27, Proposition 4.2], there exists an element $M_{t} \in \pi_{16 t+18}^{8 t+8}$ for $t \geq 0$ such that

$$
\left[\iota_{8 t+11}, \iota_{8 t+11}\right]=E^{3}\left(M_{t}\right) \text { and } H M_{t}=\nu_{16 t+15}(t \geq 0) .
$$

Hereafter, we fix $n=16 s+7 \geq 23$. By [18, there exists a lift $\left[\sigma_{n-8}\right] \in$ $\pi_{n-1}(S O(n-7))$ of $\sigma_{n-8}$. By use of the exact sequences $\left(\mathcal{S O}_{n-1}^{n-k}\right)$ for $6 \leq$ $k \leq 8$, by the fact that $\sharp \Delta \sigma_{n-7}=240, \Delta \nu_{n-6}^{2} \neq 0$ and by (4.11), we obtain the following:

$$
\pi_{n-1}(S O(n-7))=\left\{\left[\sigma_{n-8}\right], \Delta \sigma_{n-7}, \tau_{n-7}^{\prime} \nu_{n-7}^{2}\right\} \cong\left(\mathbb{Z}_{16}\right)^{2} \oplus \mathbb{Z}_{2} \oplus \mathbb{Z}_{15} .
$$

By use of $\left(\mathcal{S O}_{n-1}^{n-k}\right)$ for $1 \leq k \leq 5$ and by [4], [5], [12] and [14, we see that $\pi_{n-1}(S O(n-k))=\left\{\left(i_{n-7, n-k}\right)\left[\sigma_{n-8}\right]\right\} \cong \mathbb{Z}_{4 k}$ for $k=1,2,4$. Therefore, $\left(i_{n-7, n-1}\right)\left[\sigma_{n-8}\right]= \pm \tau_{n-1}^{\prime},\left(i_{n-7, n}\right)\left[\sigma_{n-8}\right]=\Delta \iota_{n}$ and $\gamma$ in (2.4) is taken as $\gamma=$ $J\left[\sigma_{n-8}\right]$. By (3.3) and (5.1), $\Delta\left(\eta_{n-1} \sigma_{n}\right)=2\left(\tau_{n-1}^{\prime} \sigma_{n-1}\right)$. Hence, by (1.2) and (5.1), $2\left(E^{6} \gamma\right)=\left[\iota_{n-1}, \eta_{n-1}\right]$ and $2 E^{6}\left(\gamma \sigma_{2 n-8}\right)=\left[\iota_{n-1}, \eta_{n-1} \sigma_{n}\right]$. Assume that $E^{7}\left(\gamma \sigma_{2 n-8}\right)=\left[\iota_{n}, \sigma_{n}\right]=0$. Then, by $\left(\mathcal{P} \mathcal{E}_{2 n+5}^{n-1}\right)$ and Lemma 5.2. $E^{6}\left(\gamma \sigma_{2 n-8}\right)=$ $\left[\iota_{n-1}, \bar{\nu}_{n-1}\right]=E^{6} J\left[\kappa_{n-7}\right]$. By $\left(\mathcal{P} \mathcal{E}_{2 n+4}^{n-2}\right)$, we have

$$
E^{5}\left(\gamma \sigma_{2 n-8}-J\left[\kappa_{n-7}\right]\right) \in P \pi_{2 n+6}^{2 n-3} .
$$

By Corollary 4.5 (2) and its proof, $P\left(\nu_{2 n-3}^{3}\right)=0, P \mu_{2 n-3} \neq 0$ and

$$
P\left(\eta_{2 n-3}^{2} \sigma_{2 n-1}\right)= \begin{cases}0, & \text { if } n \neq 53(\bmod 64) \\ 4 E\left(\bar{\tau}_{n-3} \sigma_{2 n-4}\right), & \text { if } n \equiv 53(\bmod 64) .\end{cases}
$$


So, for $b$ and $x \in\{0,1\}$, we have

$$
E^{5}\left(\gamma \sigma_{2 n-8}-J\left[\kappa_{n-7}\right]\right)=4 b E\left(\bar{\tau}_{n-3} \sigma_{2 n-4}\right)+x P \mu_{2 n-3} .
$$

By [31, Proposition 11.10.ii)], there exists an element $\beta \in \pi_{2 n+3}^{n-3}$ such that $P \mu_{2 n-3}=E \beta$ and $H \beta=\eta_{2 n-7} \mu_{2 n-6}$. Then, by $\left(\mathcal{P} \mathcal{E}_{2 n+3}^{n-3}\right)$, we have

$$
E^{4}\left(\gamma \sigma_{2 n-8}-J\left[\kappa_{n-7}\right]\right)-4 b \bar{\tau}_{n-3} \sigma_{2 n-4}-x \beta \in P \pi_{2 n+5}^{2 n-5} .
$$

This induces a relation $x \eta_{2 n-7} \mu_{2 n-6}=0$. Hence, $x=0$ and we can set

$$
E^{4}\left(\gamma \sigma_{2 n-8}-J\left[\kappa_{n-7}\right]\right)-4 b \bar{\tau}_{n-3} \sigma_{2 n-4}=y P\left(\eta_{2 n-5} \mu_{2 n-4}\right) \text { for } y \in\{0,1\} .
$$

Since $H \bar{\tau}_{n-3}=\nu_{2 n-7}$ and $\nu_{2 n-7} \sigma_{2 n-4}=0$, we have $\bar{\tau}_{n-3} \sigma_{2 n-4}=E \xi$ for an elements $\xi \in \pi_{2 n+2}^{n-4}$. By [31, Proposition 11.10.i)], there exists an element $\beta^{\prime} \in \pi_{2 n+2}^{n-4}$ such that $P\left(\eta_{2 n-5} \mu_{2 n-4}\right)=E \beta^{\prime}$ and $H \beta^{\prime}=\eta_{2 n-9}^{2} \mu_{2 n-7}$. So, we have

$$
E^{3}\left(\gamma \sigma_{2 n-8}-J\left[\kappa_{n-7}\right]\right)-4 b \xi-y \beta^{\prime} \in P \pi_{2 n+4}^{2 n-7} .
$$

This leads to a relation $y \eta_{2 n-9}^{2} \mu_{2 n-7}=0$, and hence $y=0$. Therefore, by (5.7), we obtain $(n=16 s+7)$

$$
E^{3}\left(\gamma \sigma_{2 n-8}-J\left[\kappa_{n-7}\right]-e M_{2 s-1} \zeta_{2 n-12}\right)-4 b \xi=0(e \in\{0,1\}) .
$$

We consider the EHP sequence

$$
\pi_{2 n+1}^{n-5} \stackrel{E}{\longrightarrow} \pi_{2 n+2}^{n-4} \stackrel{H}{\longrightarrow} \pi_{2 n+2}^{2 n-9} \stackrel{P}{\longrightarrow} \pi_{2 n}^{n-5} .
$$

By (5.6),$H \xi=4 z \zeta_{2 n-9}$ for $z \in\{0,1\}$, and so there exists an element $\xi^{\prime} \in$ $\pi_{2 n+1}^{n-5}$ satisfying $E \xi^{\prime}=2 \xi$. Since $\pi_{2 n+1}^{2 n-11}=\pi_{2 n}^{2 n-13}=0$, there exists an element $\xi^{\prime \prime} \in \pi_{2 n-1}^{n-7}$ satisfying $E^{2} \xi^{\prime \prime}=\xi^{\prime}$. Hence, we have

$$
\begin{aligned}
& E^{2}\left(\gamma \sigma_{2 n-8}-J\left[\kappa_{n-7}\right]-e M_{2 s-1} \zeta_{2 n-12}\right)-2 b \xi^{\prime} \in P \pi_{2 n+3}^{2 n-9}=0, \\
& E\left(\gamma \sigma_{2 n-8}-J\left[\kappa_{n-7}\right]-e M_{2 s-1} \zeta_{2 n-12}\right)-2 b E \xi^{\prime \prime} \in P \pi_{2 n+2}^{2 n-11}=0
\end{aligned}
$$

and

$$
\gamma \sigma_{2 n-8}-J\left[\kappa_{n-7}\right]-e M_{2 s-1} \zeta_{2 n-12}-2 b \xi^{\prime \prime} \in P \pi_{2 n+1}^{2 n-13} .
$$

We note $H\left(M_{2 s-1} \zeta_{2 n-12}\right)=\nu_{2 n-15} \zeta_{2 n-12}=0$. Then, the last relation induces a contradictory relation $\sigma_{2 n-15}^{2}=\kappa_{2 n-15}$. Thus, we obtain the non-triviality of $\left[\iota_{n}, \sigma_{n}\right]$ if $n \equiv 7(\bmod 16) \geq 23$.

By Lemma 5.2, we have $\left[\iota_{n}, \bar{\nu}_{n}\right]=E^{6} J\left[\kappa_{n-7}\right]$ if $n \equiv 6(\bmod 8) \geq 14$. By the parallel arguments to the above, we obtain

Corollary 5.4. $\left[\iota_{n}, \bar{\nu}_{n}\right] \neq 0$, if $n \equiv 6(\bmod 8) \geq 14$. 


\section{Gottlieb groups of spheres with stems for $8 \leq k \leq 13$}

We know that $\pi_{n+8}\left(\mathbb{S}^{n}\right)=\left\{\varepsilon_{n}\right\} \cong \mathbb{Z}_{2}$ for $n=4,5$ and that $\left[\iota_{4}, \varepsilon_{4}\right]=\left(E \nu^{\prime}\right) \varepsilon_{7} \neq$ $0,\left[\iota_{5}, \varepsilon_{5}\right]=\nu_{5} \eta_{8} \varepsilon_{9} \neq 0$. It is easy to show that $G_{16}\left(\mathbb{S}^{8}\right)=\left\{\left(E \sigma^{\prime}\right) \eta_{15}, \sigma_{8} \eta_{15}+\right.$ $\left.\bar{\nu}_{8}+\varepsilon_{8}\right\} \cong\left(\mathbb{Z}_{2}\right)^{2}$ and $G_{17}\left(\mathbb{S}^{9}\right)=\left\{\left[\iota_{9}, \iota_{9}\right]\right\} \cong \mathbb{Z}_{2}$. So, by Lemma 4.4, we get

$$
G_{n+8}\left(\mathbb{S}^{n}\right)=0, \quad \text { if } \quad n \equiv 0,1(\bmod 4) \geq 4 \text { unless } n=8,9
$$

Let $n \equiv 3(\bmod 4) \geq 11$. Then, by Lemma 1.2 (1) and (2.1), $\left[\iota_{n}, \eta_{n} \sigma_{n+1}\right]=$ 0 . In virtue of (1.2) and Example 3.4.(1), we obtain $\left[\iota_{n}, \varepsilon_{n}\right]=0$. Thus,

$$
G_{n+8}\left(\mathbb{S}^{n}\right)=\pi_{n+8}\left(\mathbb{S}^{n}\right), \quad \text { if } n \equiv 3(\bmod 4) .
$$

Now, we show the following

Lemma 6.1. (1) Let $n \equiv 2(\bmod 8) \geq 10$. Then $\Delta \varepsilon_{n}=0$ and $\left[\iota_{n}, \bar{\nu}_{n}\right]=$ $\left[\iota_{n}, \eta_{n} \sigma_{n+1}\right] \neq 0$.

(2) Let $n \equiv 6(\bmod 8) \geq 14$. Then $\Delta \varepsilon_{n}=2 a\left(i_{n}(\mathbb{R})\right)\left[\nu_{n-2}^{2}\right] \nu_{n+4}$ for $a \in$ $\{0,1,2,3\}$. And the order of $\Delta \pi_{n+8}\left(\mathbb{S}^{n}\right)$ is four or two according as $n \equiv$ $22(\bmod 32)$ or not.

Proof. Let $n \equiv 2(\bmod 4) \geq 10$. Then, by the fact that $\pi_{n+1}(S O(n)) \cong \mathbb{Z}$ [14, we have $\tau_{n}^{\prime} \eta_{n}=0$. So, by (3.3), (4.9) and (5.1), we obtain

$$
\Delta\left(\eta_{n} \bar{\eta}_{n+1}\right)=2 \tau_{n}^{\prime} \circ \bar{\eta}_{n}=\tau_{n}^{\prime} \circ \eta_{n}^{2} p_{n+2}=0 .
$$

Therefore, by Lemma 4.2, we get

$$
\begin{aligned}
\Delta \varepsilon_{n}=\Delta \iota_{n} \circ \varepsilon_{n-1} & =\Delta \iota_{n} \circ\left\{\eta_{n-1} \bar{\eta}_{n}, \tilde{\eta}_{n+1}, \nu_{n+3}\right\} \\
& =-\left\{\Delta \iota_{n}, \eta_{n-1} \bar{\eta}_{n}, \tilde{\eta}_{n+1}\right\} \circ \nu_{n+4} .
\end{aligned}
$$

We have

$$
\left\{\Delta \iota_{n}, \eta_{n-1} \bar{\eta}_{n}, \tilde{\eta}_{n+1}\right\} \subset \pi_{n+4}(S O(n)) .
$$

In virtue of [4, [5] and [12], $\pi_{n+4}(S O(n)) \cong \mathbb{Z}_{8 d}$, where $d=2$ or 1 according as $n \equiv 2(\bmod 8) \geq 10$ or $n \equiv 6(\bmod 8) \geq 14$. Noting the relation $4 \tilde{\eta}_{n+1}=0$, we obtain

$$
\begin{aligned}
4\left\{\Delta \iota_{n}, \eta_{n-1} \bar{\eta}_{n}, \tilde{\eta}_{n+1}\right\} & =-\Delta \iota_{n} \circ\left\{\eta_{n-1} \bar{\eta}_{n}, \tilde{\eta}_{n+1}, 4 \iota_{n+3}\right\} \\
& \subset-\Delta \iota_{n} \circ \pi_{n+4}\left(\mathbb{S}^{n-1}\right)=0 .
\end{aligned}
$$


This induces $\Delta \varepsilon_{n} \in(2 d)\left(\pi_{n+4}(S O(n)) \circ \nu_{n+4}\right)$. Since $4 \pi_{n+7}(S O(n))=0$ by [4, [5] and [12], we obtain the first assertion of (1).

Let $n \equiv 6(\bmod 8) \geq 14$. By the exact sequences $\left(\mathcal{S O}_{n+4}^{n+k}\right)$ for $k=$ $-2,-1$ and Lemma 3.1 we get that $i_{n}(\mathbb{R})_{*}: \pi_{n+4}(S O(n-1)) \rightarrow \pi_{n+4}(S O(n))$ is an isomorphism and $\pi_{n+4}(S O(n-1))=\left\{\left[\nu_{n-2}^{2}\right]\right\} \cong \mathbb{Z}_{8}$. This leads to the first assertion of (2).

We recall from [19] that $\sharp\left[\iota_{n}, \eta_{n} \sigma_{n+1}\right]=2$ if $n \equiv 2(\bmod 8) \geq 10$. So, by the first half, we obtain the second half of (1).

By (1.3) and (2.5), $\Delta: \pi_{n+7}\left(\mathbb{S}^{n}\right) \rightarrow \pi_{n+6}(S O(n))$ is a monomorphism for even $n \geq 10$. So, by $\left(\mathcal{S O}_{n+7}^{n}\right)$, we have the exact sequence:

$$
\pi_{n+8}\left(\mathbb{S}^{n}\right) \stackrel{\Delta}{\longrightarrow} \pi_{n+7}(S O(n)) \stackrel{i_{*}}{\longrightarrow} \pi_{n+7}(S O(n+1)) \longrightarrow 0 .
$$

By [4, 5] and [12, we know that

$$
\pi_{n+7}(S O(n+1)) \cong \begin{cases}\left(\mathbb{Z}_{2}\right)^{2}, & \text { if } n \equiv 6(\bmod 16) \geq 22 ; \\ \mathbb{Z}_{2}, & \text { if } n \equiv 14(\bmod 16)\end{cases}
$$

and by (4.16),

$$
\pi_{n+7}(S O(n)) \cong \begin{cases}\mathbb{Z}_{4} \oplus \mathbb{Z}_{2}, & \text { if } n \equiv 6(\bmod 32) \geq 38 \\ \left(\mathbb{Z}_{4}\right)^{2}, & \text { if } n \equiv 22(\bmod 32) ; \\ \mathbb{Z}_{4}, & \text { if } n \equiv 14(\bmod 16) .\end{cases}
$$

Hence, we obtain the second half of (2). This completes the proof.

Now, by Lemma 6.1.(1),

$$
\left[\iota_{n}, \varepsilon_{n}\right]=0 \text { and }\left[\iota_{n}, \bar{\nu}_{n}\right]=\left[\iota_{n}, \eta_{n} \sigma_{n+1}\right] \neq 0 \text {, if } n \equiv 2(\bmod 8) \geq 10 .
$$

Whence, we conclude that

$$
G_{n+8}\left(\mathbb{S}^{n}\right)=\left\{\varepsilon_{n}\right\} \cong \mathbb{Z}_{2}, \text { if } n \equiv 2(\bmod 8) \geq 10 .
$$

Next, by (1.3) and Lemma 6.1.(2), we obtain

$$
G_{n+8}\left(\mathbb{S}^{n}\right) \neq 0 \text {, if } n \equiv 6(\bmod 8) \geq 14 \text { unless } n \equiv 22(\bmod 32) .
$$

By [22], [28] and [31, we obtain $G_{14}\left(\mathbb{S}^{6} ; 2\right)=\pi_{14}^{6}$ and $G_{n+8}\left(\mathbb{S}^{n}\right)=\left\{\eta_{n} \sigma_{n+1}\right\} \cong$ $\mathbb{Z}_{2}$ if $n=14,22$. Since $\left[\iota_{6},\left[\iota_{6}, \alpha_{1}(6)\right]\right]=0$ by Proposition 1.5, we obtain $G_{14}\left(\mathbb{S}^{6} ; 3\right)=\pi_{14}\left(\mathbb{S}^{6} ; 3\right)$. Thus, we have shown 
Proposition 6.2. The group $G_{n+8}\left(\mathbb{S}^{n}\right)$ is equal to the following group: 0 if $n \equiv 0,1(\bmod 4) \geq 4$ unless $n=8,9 ; \pi_{n+8}\left(\mathbb{S}^{n}\right)$ if $n=6$ or $n \equiv 3(\bmod 4)$; $\left\{\varepsilon_{n}\right\} \cong \mathbb{Z}_{2}$, if $n \equiv 2(\bmod 8) \geq 10$. Moreover, $G_{n+8}\left(\mathbb{S}^{n}\right) \neq 0$ if $n \equiv$ $6(\bmod 8) \geq 14$ unless $n \equiv 22(\bmod 32), G_{16}\left(\mathbb{S}^{8}\right)=\left\{\left(E \sigma^{\prime}\right) \eta_{15}, \sigma_{8} \eta_{15}+\bar{\nu}_{8}+\right.$ $\left.\varepsilon_{8}\right\} \cong\left(\mathbb{Z}_{2}\right)^{2}, G_{17}\left(\mathbb{S}^{9}\right)=\left\{\left[\iota_{9}, \iota_{9}\right]\right\} \cong \mathbb{Z}_{2}$ and $G_{n+8}\left(\mathbb{S}^{n}\right)=\left\{\eta_{n} \sigma_{n+1}\right\} \cong \mathbb{Z}_{2}$ if $n=14,22$.

Finally, we propose

Conjecture 6.3. $\left[\iota_{n}, \eta_{n} \sigma_{n+1}\right]=0$ and $G_{n+8}\left(\mathbb{S}^{n}\right)=\left\{\eta_{n} \sigma_{n+1}\right\} \cong \mathbb{Z}_{2}$, if $n \equiv$ $6(\bmod 8) \geq 14$.

Obviously, we obtain $G_{15}\left(\mathbb{S}^{6}\right)=\pi_{15}\left(\mathbb{S}^{6}\right)$ and $G_{19}\left(\mathbb{S}^{10}\right)=\left\{3\left[\iota_{10}, \iota_{10}\right], \nu_{10}^{3}, \eta_{10} \varepsilon_{11}\right\} \cong$ $3 \mathbb{Z} \oplus\left(\mathbb{Z}_{2}\right)^{2}$. Let $n \equiv 2(\bmod 4) \geq 14$. Then, by (5.1),

$$
\left[\iota_{n}, \eta_{n}^{2} \sigma_{n+2}\right]=\left[\iota_{n}, \eta_{n} \varepsilon_{n+1}\right]=0 .
$$

By (4.13), $\left[\iota_{n}, \mu_{n}\right] \neq 0$. Whence, we obtain

$$
G_{n+9}\left(\mathbb{S}^{n}\right)=\left\{\nu_{n}^{3}, \eta_{n} \varepsilon_{n+1}\right\} \cong\left(\mathbb{Z}_{2}\right)^{2}, \text { if } n \equiv 2(\bmod 4) \geq 14 .
$$

Let now $n \equiv 3(\bmod 4) \geq 11$. Then, by Lemma 1.2(1) and (2.1), $\left[\iota_{n}, \eta_{n}^{2} \sigma_{n+2}\right]=\left[\iota_{n}, \eta_{n} \varepsilon_{n+1}\right]=0$ and by Example [3.4. $(2),\left[\iota_{n}, \mu_{n}\right]=0$. Whence, we obtain

$$
G_{n+9}\left(\mathbb{S}^{n}\right)=\pi_{n+9}\left(\mathbb{S}^{n}\right) \text {, if } n \equiv 3(\bmod 4) .
$$

It is easily seen that $G_{13}\left(\mathbb{S}^{4}\right)=\left\{\nu_{4}^{3}\right\} \cong \mathbb{Z}_{2}$. Let $n \equiv 4(\bmod 8) \geq 12$. By Lemma 1.2. (1) and (3.6), we have $\left[\iota_{n}, \nu_{n}^{3}\right]=0$. In the light of (4.13) and (4.15),$\left[\iota_{n}, \eta_{n} \varepsilon_{n+1}\right]=\left[\iota_{n}, \eta_{n}^{2} \sigma_{n+2}\right] \neq 0$ and $\left[\iota_{n}, \mu_{n}\right] \neq 0$. Assume that $P\left(\alpha_{2 n+1}+\mu_{2 n+1}\right)=0$ for $\alpha_{2 n+1}=\eta_{2 n+1} \varepsilon_{2 n+2}$ or $\eta_{2 n+1}^{2} \sigma_{2 n+3}$. By 31, Proposition 11.10.i)], there exists an element $\beta \in \pi_{2 n+7}^{n-1}$ satisfying $E \beta=0$ and $H \beta=\eta_{2 n-3}\left(\alpha_{2 n-2}+\mu_{2 n-2}\right)=\eta_{2 n-3} \mu_{2 n-2}$. On the other hand, $\left(\mathcal{P} \mathcal{E}_{2 n+7}^{n-1}\right)$ and Lemma 3.5 imply a contradictory relation $H \beta=0$. So, $\left[\iota_{n}, \alpha_{n}\right] \neq\left[\iota_{n}, \mu_{n}\right]$ and hence

$$
G_{n+9}\left(\mathbb{S}^{n}\right)=\left\{\nu_{n}^{3}\right\} \cong \mathbb{Z}_{2}, \quad \text { if } n \equiv 4(\bmod 8) .
$$

Obviously, we obtain $G_{18}\left(\mathbb{S}^{9}\right)=\left\{\sigma_{9} \eta_{16}^{2}, \nu_{9}^{3}, \eta_{9} \varepsilon_{10}\right\} \cong\left(\mathbb{Z}_{2}\right)^{3}$. Let now $n \equiv$ $1(\bmod 8) \geq 17$. By (4.13), $\left[\iota_{n}, \mu_{n}\right] \neq 0$ and by (4.17), $\left[\iota_{n}, \eta_{n} \varepsilon_{n+1}\right]=0$. In the light of IV, $\left[\iota_{n}, \eta_{n}^{2} \sigma_{n+2}\right]=0$ if $n=2^{i}-7$ for $i \geq 4$ and $\left[\iota_{n}, \nu_{n}^{3}\right]=$ $\left[\iota_{n}, \eta_{n}^{2} \sigma_{n+2}\right] \neq 0$ if $n \equiv 1(\bmod 8) \geq 17$ and $n \neq 2^{i}-7$. By the parallel 
argument to the case $n \equiv 4(\bmod 8)$, we get $\left[\iota_{n}, \eta_{n}^{2} \sigma_{n+2}\right] \neq\left[\iota_{n}, \mu_{n}\right]$ [31, Proposition 11.10.ii)]. So, we obtain

$$
G_{n+9}\left(\mathbb{S}^{n}\right)=\left\{\begin{array}{l}
\left\{\eta_{n} \varepsilon_{n+1}\right\} \cong \mathbb{Z}_{2}, \text { if } n \equiv 1(\bmod 8) \geq 17 \text { and } n \neq 2^{i}-7 \\
\left\{\eta_{n} \varepsilon_{n+1}, \eta_{n}^{2} \sigma_{n+2}\right\} \cong\left(\mathbb{Z}_{2}\right)^{2}, \text { if } n=2^{i}-7(i \geq 5) .
\end{array}\right.
$$

Obviously, we obtain $G_{14}\left(\mathbb{S}^{5}\right)=\left\{\nu_{5}^{3}, \eta_{5} \varepsilon_{6}\right\} \cong\left(\mathbb{Z}_{2}\right)^{2}$. Let $n \equiv 5(\mathrm{mod}$ $8) \geq 13$. By Corollary 4.5. (2) and (4.13), $\nu_{n}^{3} \in G_{n+9}\left(\mathbb{S}^{n}\right)$ and $\mu_{n} \notin G_{n+9}\left(\mathbb{S}^{n}\right)$. Furthermore, by Corollary 4.5. $(2), \eta_{n} \varepsilon_{n+1} \in G_{n+9}\left(\mathbb{S}^{n}\right)$ unless $n \equiv 53(\bmod$ 64). So, we obtain

$G_{n+9}\left(\mathbb{S}^{n}\right)=\left\{\nu_{n}^{3}, \eta_{n} \varepsilon_{n+1}\right\} \cong\left(\mathbb{Z}_{2}\right)^{2}$, if $n \equiv 5(\bmod 8)$ and $n \not \equiv 53(\bmod 64)$.

At the end, we use the following:

$\zeta_{n} \in\left\{2 \iota_{n}, \eta_{n}, \alpha_{n+1}\right\}_{2}\left(\bmod 2 \zeta_{n}\right)$ for $\alpha_{n+1}=\eta_{n+1}^{2} \sigma_{n+3}$ or $\eta_{n+1} \varepsilon_{n+2}$, if $n \geq 11$.

Let $n \equiv 0(\bmod 8) \geq 16$. By [31, Proposition 11.11.i)], there exists an element $\beta \in \pi_{2 n+6}^{n-2}$ such that $\left[\iota_{n}, \alpha_{n}\right]=E^{2} \beta$ and $H \beta \in\left\{2 \iota_{2 n-5}, \eta_{2 n-5}\right.$, $\left.\alpha_{2 n-4}\right\}_{2} \ni \zeta_{2 n-5}\left(\bmod 2 \zeta_{2 n-5}\right)$. Assume that $\left[\iota_{n}, \alpha_{n}\right]=0$. Then, $\left(\mathcal{P} \mathcal{E}_{2 n+7}^{n-1}\right)$ and (2.4) induce a relation $E\left(\beta-a E^{6}\left(\gamma \eta_{2 n-10} \mu_{2 n-9}\right)\right)=0$ for $a \in\{0,1\}$. Hence, by $\left(\mathcal{P} \mathcal{E}_{2 n+6}^{n-2}\right)$ and Lemma 3.5, we have a contradictory relation $\zeta_{2 n-5} \in$ $2 \pi_{2 n+6}^{2 n-5}$. Whence, we get that $\left[\iota_{n}, \alpha_{n}\right] \neq 0$. In the light of (4.13) and (4.14), we know $\left[\iota_{n}, \mu_{n}\right] \neq 0$ and $\left[\iota_{n}, \mu_{n}\right] \eta_{2 n+8} \neq 0$. This implies that $\left[\iota_{n}, \alpha_{n}\right] \neq$ $\left[\iota_{n}, \mu_{n}\right]$ and $\left[\iota_{n}, \nu_{n}^{3}\right] \neq\left[\iota_{n}, \mu_{n}\right]$. It is easy to show that $\left[\iota_{8}, \nu_{8}^{3}\right]=\eta_{8} \bar{\varepsilon}_{9}$ and $G_{17}\left(\mathbb{S}^{8}\right)=\left\{\left(E \sigma^{\prime}\right) \eta_{15}^{2}, \sigma_{8} \eta_{15}^{2}+\nu_{8}^{3}+\eta_{8} \varepsilon_{9}\right\} \cong\left(\mathbb{Z}_{2}\right)^{2}$. By [23] and [31], we obtain $G_{25}\left(\mathbb{S}^{16}\right)=0$. By [26, 4.14], there exists an element $\tau_{1} \in \pi_{2 n+2}^{n-6}$ such that

$$
\left[\iota_{n}, \nu_{n}^{3}\right]=E^{6} \tau_{1}, H \tau_{1}=\eta_{2 n-13} \kappa_{2 n-12}, \text { if } n \equiv 0(\bmod 8) \geq 16 .
$$

Assume that $\left[\iota_{n}, \nu_{n}^{3}\right]=0$. Then, by $\left(\mathcal{P} \mathcal{E}_{2 n+7}^{n-1}\right)$ and $(\underline{2.4})$, we have $E^{5}\left(\tau_{1}-\right.$ $\left.a E^{2}\left(\gamma \eta_{2 n-10} \mu_{2 n-9}\right)\right)=0$ for $a \in\{0,1\}$. So, by $\left(\mathcal{P} \mathcal{E}_{2 n+6}^{n-2}\right)$, we have $E^{4}\left(\tau_{1}-\right.$ $\left.a E^{2}\left(\gamma \eta_{2 n-10} \mu_{2 n-9}\right)\right) \in P \pi_{2 n+8}^{2 n-3}=\left\{\left[\iota_{n-2}, \zeta_{n-2}\right]\right\}$. By applying $H: \pi_{2 n+6}^{n-2} \rightarrow$ $\pi_{2 n+6}^{2 n-5}$ to this relation and by (5.6) $), E^{4}\left(\tau_{1}-a E^{2}\left(\gamma \eta_{2 n-10} \mu_{2 n-9}\right)\right)=0$. By the fact that $\pi_{2 n+7}^{2 n-5}=\pi_{2 n+6}^{2 n-7}=0$, we obtain $E^{2}\left(\tau_{1}-a E^{2}\left(\gamma \eta_{2 n-10} \mu_{2 n-9}\right)\right)=0$. Hence, by $\left(\mathcal{P} \mathcal{E}_{2 n+3}^{n-5}\right)$ and (5.7), we have

$$
E\left(\tau_{1}-a E^{2}\left(\gamma \eta_{2 n-10} \mu_{2 n-9}\right)\right) \in P \pi_{2 n+5}^{2 n-9}=E^{3} M_{t} \circ\left\{\sigma_{2 n-11}^{2}, \kappa_{2 n-11}\right\}
$$

for $n=8 t+16$. By $\left(\mathcal{P} \mathcal{E}_{2 n+2}^{n-6}\right)$, we obtain

$$
\tau_{1}-E^{2}\left(a \gamma \eta_{2 n-10} \mu_{2 n-9}+b M_{t} \sigma_{2 n-14}^{2}+c M_{t} \kappa_{2 n-14}\right) \in P \pi_{2 n+4}^{2 n-11}
$$


with $b, c \in\{0,1\}$. This induces a contradictory relation $\eta_{2 n-13} \kappa_{2 n-12} \in$ $2 \pi_{2 n+2}^{2 n-13}$. Thus, we conclude that

$$
\left[\iota_{n}, \nu_{n}^{3}\right] \neq 0, \text { if } n \equiv 0(\bmod 8) \geq 16
$$

Summing the above, we get

Proposition 6.4. The group $G_{n+9}\left(\mathbb{S}^{n}\right)$ is equal to the following group: $\pi_{n+9}\left(\mathbb{S}^{n}\right)$ if $n=6$ or $n \equiv 3(\bmod 4) ;\left\{\nu_{n}^{3}, \eta_{n} \varepsilon_{n+1}\right\} \cong\left(\mathbb{Z}_{2}\right)^{2}$ if $n \equiv 2(\bmod$ 4) $\geq 14, n=2^{i}-7$ for $i \geq 5$ or $n \equiv 5(\bmod 8)$ unless $n \equiv 53(\bmod 64)$; $\left\{\nu_{n}^{3}\right\} \cong \mathbb{Z}_{2}$ if $n \equiv 4(\bmod 8) ;\left\{\eta_{n} \varepsilon_{n+1}\right\} \cong \mathbb{Z}_{2}$ if $n \equiv 1(\bmod 8) \geq 17$ and $n \neq 2^{i}-7 ; 0$ if $n \equiv 0(\bmod 8) \geq 16$. Moreover, $G_{17}\left(\mathbb{S}^{8}\right)=\left\{\left(E \sigma^{\prime}\right) \eta_{15}^{2}, \sigma_{8} \eta_{15}^{2}+\right.$ $\left.\nu_{8}^{3}+\eta_{8} \varepsilon_{9}\right\} \cong\left(\mathbb{Z}_{2}\right)^{2}, G_{18}\left(\mathbb{S}^{9}\right)=\left\{\sigma_{9} \eta_{16}^{2}, \nu_{9}^{3}, \eta_{9} \varepsilon_{10}\right\} \cong\left(\mathbb{Z}_{2}\right)^{3}$ and $G_{19}\left(\mathbb{S}^{10}\right)=$ $\left\{3\left[\iota_{10}, \iota_{10}\right], \nu_{10}^{3}, \eta_{10} \varepsilon_{11}\right\} \cong 3 \mathbb{Z} \oplus\left(\mathbb{Z}_{2}\right)^{2}$.

By Lemma 1.1. Corollary 1.3.(3), Proposition 1.4 and (4.14), we have determined $G_{n+10}\left(\mathbb{S}^{n}\right)$ for $n \geq 12$.

It is easily seen that

$$
G_{n+10}\left(\mathbb{S}^{n}\right)= \begin{cases}\left\{\nu_{4} \sigma^{\prime}+E \varepsilon^{\prime}, 2 E \varepsilon^{\prime}, \alpha_{1}(4) \alpha_{2}(7),\right. & \text { if } n=4 ; \\ \left.\nu_{4} \alpha_{2}(7), \nu_{4} \alpha_{1}^{\prime}(7)\right\}, & \text { if } n=5 ; \\ \pi_{15}\left(\mathbb{S}^{5}\right), & \text { if } n=6 ; \\ \pi_{16}^{6} \oplus \mathbb{Z}_{3}\left\{3 \beta_{1}(6)\right\}, & \text { if } n=8 ; \\ \left\{\sigma_{8} \nu_{15}, \nu_{8} \sigma_{11}, \sigma_{8} \alpha_{1}(15)\right\}, & \text { if } n=9 ; \\ \left\{\sigma_{9} \nu_{16}, \beta_{1}(9)\right\}, & \text { if } n=10 \\ \pi_{20}^{10}=\left\{\sigma_{10} \nu_{17}, \eta_{10} \mu_{11}\right\}, & \text { if } n=11 \\ \pi_{21}\left(\mathbb{S}^{11}\right), & \end{cases}
$$

Thus, by summing up the above results, we get

Proposition 6.5. The group $G_{n+10}\left(\mathbb{S}^{n}\right)$ is isomorphic to one of the following groups: $\mathbb{Z}_{120} \oplus \mathbb{Z}_{6}, \mathbb{Z}_{72} \oplus \mathbb{Z}_{2}, \mathbb{Z}_{24} \oplus \mathbb{Z}_{2}, \mathbb{Z}_{24} \oplus \mathbb{Z}_{8}, \mathbb{Z}_{24}, \mathbb{Z}_{4} \oplus \mathbb{Z}_{2}, \mathbb{Z}_{6} \oplus \mathbb{Z}_{2}$ according as $n=4,5,6,8,9,10,11$. Furthermore, $G_{n+10}\left(\mathbb{S}^{n}\right)$ is isomorphic to the group: 0 if $n \equiv 0(\bmod 4) \geq 12 ; \mathbb{Z}_{2}$ if $n \equiv 2(\bmod 4) \geq 14 ; \mathbb{Z}_{3}$ if $n \equiv 1(\bmod 4) \geq 13$ and $\mathbb{Z}_{6}$ if $n \equiv 3(\bmod 4) \geq 15$.

We recall that $\pi_{n+11}\left(\mathbb{S}^{n} ; 3\right)=\left\{\alpha_{3}(n)\right\} \cong \mathbb{Z}_{3}$ for $n=3,4$ and that $\pi_{n+11}\left(\mathbb{S}^{n} ; 3\right)=\left\{\alpha_{3}^{\prime}(n)\right\} \cong \mathbb{Z}_{9}$ for $n \geq 5$, where $3 \alpha_{3}^{\prime}(n)=\alpha_{3}(n)$ for $n \geq 5$. By [22], 23], 24], 31] and (5.6) $, \sharp\left[\iota_{n}, \zeta_{n}\right]=1,4,8,1,4,1,8,1$ according as 
$n=5,6,8,9,10,11,12,13$. We easily obtain that

$$
G_{n+11}\left(\mathbb{S}^{n}\right)= \begin{cases}\left\{\nu_{4} \sigma^{\prime} \eta_{14}, \nu_{4} \bar{\nu}_{7}, \nu_{4} \varepsilon_{7},\right. & \\ \left.2 E \mu^{\prime}, \varepsilon_{4} \nu_{12},\left(E \nu^{\prime}\right) \varepsilon_{7}\right\}, & \text { if } n=4 ; \\ \pi_{16}\left(\mathbb{S}^{5}\right), & \text { if } n=5 \\ \left\{4 \zeta_{6}, \bar{\nu}_{6} \nu_{14}\right\}, & \text { if } n=6 \\ \left\{\bar{\nu}_{8} \nu_{16}\right\}, & \text { if } n=8 \\ \pi_{20}\left(\mathbb{S}^{9}\right), & \text { if } n=9 \\ 4 \pi_{21}^{10}, & \text { if } n=10 \\ \pi_{22}\left(\mathbb{S}^{11}\right), & \text { if } n=11 \\ \left\{3\left[\iota_{12}, \iota_{12}\right]\right\}, & \text { if } n=12\end{cases}
$$

By abuse of notations, $\zeta_{n}$ for $n \geq 5$ represents a generator of the direct summands $\mathbb{Z}_{8}$ of $\pi_{n+11}^{n}$ and $\mathbb{Z}_{504}$ of $\pi_{n+11}\left(\mathbb{S}^{n}\right)$, respectively. By [19], 222], 22], 31, Corollary 1.3. (3), Proposition 1.4 and (5.6), we obtain

$$
\sharp\left[\iota_{n}, \zeta_{n}\right]= \begin{cases}1, & \text { if } n \equiv 1,5,7(\bmod 8) \geq 5 ; \\ 252, & \text { if } n \equiv 2(\bmod 4) \geq 6 ; \\ 504, & \text { if } n \equiv 0(\bmod 4) \geq 8 .\end{cases}
$$

Assume that $n \equiv 3(\bmod 8) \geq 19$. Then, by [4], [5] and [12], we obtain

$$
\pi_{n+10}(S O(n)) \cong \begin{cases}\left(\mathbb{Z}_{2}\right)^{3}, & \text { if } n \equiv 3(\bmod 32) \geq 35 ; \\ \left(\mathbb{Z}_{2}\right)^{2} \oplus \mathbb{Z}_{4}, & \text { if } n \equiv 19(\bmod 64) ; \\ \left(\mathbb{Z}_{2}\right)^{2} \oplus \mathbb{Z}_{8}, & \text { if } n \equiv 51(\bmod 128) ; \\ \left(\mathbb{Z}_{2}\right)^{2} \oplus \mathbb{Z}_{16}, & \text { if } n \equiv 115(\bmod 128) ; \\ \left(\mathbb{Z}_{2}\right)^{2}, & \text { if } n \equiv 11(\bmod 16) \geq 27\end{cases}
$$

and

$$
\pi_{n+10}(S O(n+1)) \cong \begin{cases}\left(\mathbb{Z}_{2}\right)^{4} \oplus \mathbb{Z}_{3}, & \text { if } n \equiv 3(\bmod 32) \geq 35 \\ \left(\mathbb{Z}_{2}\right)^{3} \oplus \mathbb{Z}_{12}, & \text { if } n \equiv 19(\bmod 64) ; \\ \left(\mathbb{Z}_{2}\right)^{3} \oplus \mathbb{Z}_{24}, & \text { if } n \equiv 51(\bmod 64) ; \\ \left(\mathbb{Z}_{2}\right)^{3} \oplus \mathbb{Z}_{3}, & \text { if } n \equiv 11(\bmod 16) \geq 27\end{cases}
$$

In the exact sequence $\left(\mathcal{S O}_{n+10}^{n}\right)$, we get $p_{n+1}(\mathbb{R})_{*}\left(\left[\eta_{n}\right] \mu_{n+1}\right)=\eta_{n} \mu_{n+1}$ and $p_{n+1}(\mathbb{R})_{*}\left[\beta_{1}(n)\right]=\beta_{1}(n)$. So, $p_{n+1}(\mathbb{R})_{*}$ is a split epimorphism. Whence, by the group structures of $\pi_{n+10}(S O(n+k))$ for $k=0,1$, we obtain

$$
\Delta \zeta_{n}=0 \text {, if } n \not \equiv 115(\bmod 128) \text { and } \Delta \zeta_{n} \neq 0 \text {, if } n \equiv 115(\bmod 128) \text {. }
$$


So, we have $\left[\iota_{n}, \zeta_{n}\right]=0$, if $n \equiv 3(\bmod 8) \geq 19$ and $n \not \equiv 115(\bmod 128)$. Consequently, by use of Lemma 1.1] and [31], the groups $G_{n+11}\left(\mathbb{S}^{n}\right)$ have been determined if $n \geq 13$ except $n \equiv 115(\bmod 128)$. Thus, by summing up the above results, we get

Proposition 6.6. The group $G_{n+11}\left(\mathbb{S}^{n}\right)$ is isomorphic to one of the following groups: $\left(\mathbb{Z}_{2}\right)^{6}, \mathbb{Z}_{504} \oplus\left(\mathbb{Z}_{2}\right)^{2}, \mathbb{Z}_{2} \oplus \mathbb{Z}_{4}, \mathbb{Z}_{2}, \mathbb{Z}_{504} \oplus \mathbb{Z}_{2}, \mathbb{Z}_{2}, \mathbb{Z}_{504}, 3 \mathbb{Z}$ according as $n=4,5,6,8,9,10,11,12$. Furthermore, $G_{n+11}\left(\mathbb{S}^{n}\right)$ is isomorphic to the group: $\mathbb{Z}_{504}$ if $n \equiv 1,5,7(\bmod 8) \geq 13 ; \mathbb{Z}_{2}$ if $n \equiv 2(\bmod 4) \geq 14$; 0 if $n \equiv 0(\bmod 4) \geq 16$ and $\mathbb{Z}_{504}$ if $n \equiv 3(\bmod 8) \geq 19$ provided $n \not \equiv 115(\bmod 128)$.

We recall that $\zeta_{n} \in\left\{2 \iota_{n}, \eta_{n}^{3}, \sigma_{n+3}\right\}$ for $n \geq 11$. So, by the fact that $2 \Delta \iota_{n}=0$ for $n$ odd, we obtain

$$
\Delta \zeta_{n}=-\left\{\Delta \iota_{n}, 2 \iota_{n-1}, \eta_{n-1}^{3}\right\} \circ \sigma_{n+3} \text { for } n \text { odd and } n \geq 11
$$

We see that $2\left\{\Delta \iota_{n}, 2 \iota_{n-1}, \eta_{n-1}^{3}\right\}=-\Delta \iota_{n} \circ\left\{2 \iota_{n-1}, \eta_{n-1}^{3}, 2 \iota_{n+2}\right\}=0$. Hence, by the fact that $\pi_{n+3}(S O(n)) \cong \mathbb{Z}_{16}$ for $n \equiv 3(\bmod 8) \geq 11$, we obtain

Remark 6.7 $\Delta \zeta_{n} \in 8\left(\pi_{n+3}(S O(n)) \circ \sigma_{n+3}\right)$ if $n \equiv 3(\bmod 8) \geq 11$.

Finally, we recall $\pi_{22}\left(\mathbb{S}^{10}\right)=\left\{\left[\iota_{10}, \nu_{10}\right]\right\} \cong \mathbb{Z}_{12}$. By Proposition 1.5. $G_{22}\left(\mathbb{S}^{10}\right)=$ $\pi_{22}^{10}$. It is easily seen, in the light of [31], Corollary 1.3] (3) and Proposition 1.4. that $G_{n+12}\left(\mathbb{S}^{n}\right)=\pi_{n+12}\left(\mathbb{S}^{n}\right)$ unless $n=10$ and

$$
G_{n+13}\left(\mathbb{S}^{n}\right)= \begin{cases}\left\{\nu_{4}^{2} \sigma_{10}, \nu_{4} \eta_{7} \mu_{8},\left(E \nu^{\prime}\right) \eta_{7} \mu_{8},\right. & \\ \left.\left(\alpha_{1}(4)+\nu_{4}\right) \beta_{1}(7)\right\} \cong \mathbb{Z}_{24} \oplus\left(\mathbb{Z}_{2}\right)^{2}, & \text { if } n=4 \\ \left\{3\left[\iota_{14}, \iota_{14}\right]\right\} \cong 3 \mathbb{Z}, & \text { if } n=14 \\ \pi_{n+13}^{n}, & \text { if } n \text { is even } \\ & \text { unless } n=2,4,14 \\ \pi_{n+13}\left(\mathbb{S}^{n}\right), & \text { if } n \text { is odd. }\end{cases}
$$

We close the paper with the table of $G_{n+k}\left(\mathbb{S}^{n}\right)$ for $1 \leq k \leq 13$ and $2 \leq n \leq 26$ : 


\begin{tabular}{||c||c|c|c|c|c|c|c||}
\hline \hline$G_{n+k}\left(\mathbb{S}^{n}\right)$ & $\mathrm{n}=2$ & $\mathrm{n}=3$ & $\mathrm{n}=4$ & $\mathrm{n}=5$ & $\mathrm{n}=6$ & $\mathrm{n}=7$ & $\mathrm{n}=8$ \\
\hline \hline $\mathrm{k}=1$ & $\infty$ & 2 & 0 & 0 & 2 & 2 & 0 \\
\hline $\mathrm{k}=2$ & 2 & 2 & 0 & 2 & 2 & 2 & 0 \\
\hline $\mathrm{k}=3$ & 2 & 12 & $3 \infty+2$ & 24 & 2 & 24 & 0 \\
\hline $\mathrm{k}=4$ & 12 & 2 & $(2)^{2}$ & 2 & 0 & 0 & 0 \\
\hline $\mathrm{k}=5$ & 2 & 2 & $(2)^{2}$ & 2 & $3 \infty$ & 0 & 0 \\
\hline $\mathrm{k}=6$ & 2 & 3 & $24+3$ & 2 & 0 & 2 & 0 \\
\hline $\mathrm{k}=7$ & 3 & 15 & 0 & 30 & 0 & 120 & $3 \infty+2$ \\
\hline $\mathrm{k}=8$ & 15 & 2 & 0 & 0 & $24+2$ & $(2)^{3}$ & $(2)^{2}$ \\
\hline $\mathrm{k}=9$ & 2 & $(2)^{2}$ & 2 & $(2)^{2}$ & $(2)^{3}$ & $(2)^{4}$ & $(2)^{2}$ \\
\hline $\mathrm{k}=10$ & $(2)^{2}$ & $12+2$ & $120+6$ & $72+2$ & $24+2$ & $24+2$ & $24+8$ \\
\hline $\mathrm{k}=11$ & $12+2$ & $84+(2)^{2}$ & $(2)^{6}$ & $504+(2)^{2}$ & $4+2$ & $504+2$ & 2 \\
\hline $\mathrm{k}=12$ & $84+(2)^{2}$ & $(2)^{2}$ & $(2)^{6}$ & $(2)^{3}$ & 240 & 0 & 0 \\
\hline $\mathrm{k}=13$ & $(2)^{2}$ & 6 & $24+(2)^{2}$ & $6+2$ & 2 & 6 & $(2)^{2}$ \\
\hline \hline
\end{tabular}

\begin{tabular}{||c||c|c|c|c|c|c|c|c|c||}
\hline \hline$G_{n+k}\left(\mathbb{S}^{n}\right)$ & $\mathrm{n}=9$ & $\mathrm{n}=10$ & $\mathrm{n}=11$ & $\mathrm{n}=12$ & $\mathrm{n}=13$ & $\mathrm{n}=14$ & $\mathrm{n}=15$ & $\mathrm{n}=16$ & $\mathrm{n}=17$ \\
\hline \hline $\mathrm{k}=1$ & 0 & 0 & 2 & 0 & 0 & 0 & 2 & 0 & 0 \\
\hline $\mathrm{k}=2$ & 0 & 2 & 2 & 0 & 0 & 2 & 2 & 0 & 0 \\
\hline $\mathrm{k}=3$ & 12 & 2 & 12 & 2 & 24 & 2 & 24 & 0 & 12 \\
\hline $\mathrm{k}=4$ & 0 & 0 & 0 & 0 & 0 & 0 & 0 & 0 & 0 \\
\hline $\mathrm{k}=5$ & 0 & 0 & 0 & 0 & 0 & 0 & 0 & 0 & 0 \\
\hline $\mathrm{k}=6$ & 0 & 0 & 2 & 2 & 2 & 0 & 2 & 0 & 0 \\
\hline $\mathrm{k}=7$ & 120 & 0 & 240 & 0 & 120 & 0 & 240 & 0 & 120 \\
\hline $\mathrm{k}=8$ & 2 & 2 & $(2)^{2}$ & 0 & 0 & 2 & $(2)^{2}$ & 0 & 0 \\
\hline $\mathrm{k}=9$ & $(2)^{3}$ & $3 \infty+(2)^{2}$ & $(2)^{3}$ & 2 & $(2)^{2}$ & $(2)^{2}$ & $(2)^{3}$ & 0 & 2 \\
\hline $\mathrm{k}=10$ & 24 & $4+2$ & $6+2$ & 0 & 3 & 2 & 6 & 0 & 3 \\
\hline $\mathrm{k}=11$ & $504+2$ & 2 & 504 & $3 \infty$ & 504 & 2 & 504 & 0 & 504 \\
\hline $\mathrm{k}=12$ & 0 & 4 & 2 & $(2)^{2}$ & 2 & 0 & 0 & 0 & 0 \\
\hline $\mathrm{k}=13$ & 6 & 2 & $6+2$ & $(2)^{2}$ & 6 & $3 \infty$ & 3 & 0 & 3 \\
\hline \hline
\end{tabular}




\begin{tabular}{||c||c|c|c|c|c|c|c|c|c||}
\hline \hline$G_{n+k}\left(\mathbb{S}^{n}\right)$ & $\mathrm{n}=18$ & $\mathrm{n}=19$ & $\mathrm{n}=20$ & $\mathrm{n}=21$ & $\mathrm{n}=22$ & $\mathrm{n}=23$ & $\mathrm{n}=24$ & $\mathrm{n}=25$ & $\mathrm{n}=26$ \\
\hline \hline $\mathrm{k}=1$ & 0 & 2 & 0 & 0 & 0 & 2 & 0 & 0 & 0 \\
\hline $\mathrm{k}=2$ & 2 & 2 & 0 & 0 & 2 & 2 & 0 & 0 & 2 \\
\hline $\mathrm{k}=3$ & 2 & 12 & 0 & 12 & 2 & 24 & 0 & 12 & 2 \\
\hline $\mathrm{k}=4$ & 0 & 0 & 0 & 0 & 0 & 0 & 0 & 0 & 0 \\
\hline $\mathrm{k}=5$ & 0 & 0 & 0 & 0 & 0 & 0 & 0 & 0 & 0 \\
\hline $\mathrm{k}=6$ & 0 & 0 & 2 & 2 & 0 & 2 & 0 & 0 & 0 \\
\hline $\mathrm{k}=7$ & 0 & 120 & 0 & 120 & 0 & 120 & 0 & 120 & 0 \\
\hline $\mathrm{k}=8$ & 2 & $(2)^{2}$ & 0 & 0 & 2 & $(2)^{2}$ & 0 & 0 & 2 \\
\hline $\mathrm{k}=9$ & $(2)^{2}$ & $(2)^{3}$ & 2 & $(2)^{2}$ & $(2)^{2}$ & $(2)^{3}$ & 0 & 2 & $(2)^{2}$ \\
\hline $\mathrm{k}=10$ & 2 & 6 & 0 & 3 & 2 & 6 & 0 & 3 & 2 \\
\hline $\mathrm{k}=11$ & 2 & 504 & 0 & 504 & 2 & 504 & 0 & 504 & 2 \\
\hline $\mathrm{k}=12$ & 0 & 0 & 0 & 0 & 0 & 0 & 0 & 0 & 0 \\
\hline $\mathrm{k}=13$ & 0 & 3 & 0 & 3 & 0 & 3 & 0 & 3 & 0 \\
\hline \hline
\end{tabular}

Like in 31, an integer $n$ indicates the cyclic group $\mathbb{Z}_{n}$ of order $n$, the symbol $\infty$ an infinite cyclic group $\mathbb{Z}$, the symbol + the direct sum of groups and $(2)^{k}$ indicates the direct sum of $k$-copies of $\mathbb{Z}_{2}$.

\section{References}

[1] J.F. Adams, Vector fields on spheres, Ann. of Math. 75 (1962), 603-632.

[2] W.D. Barcus and M.G. Barratt, On the homotopy classification of the extensions of a fixed map, Trans. Amer. Math. Soc. 88 (1958), 57-74.

[3] M.G. Barratt, Note on a formula due to Toda, J. London Math. Soc. 36 (1661), 95-96.

[4] M.G. Barratt and M.E. Mahowald, The metastable homotopy of $O(n)$, Bull. Amer. Math. Soc. 70 (1964), 758-760.

[5] R. Bott, The stable homotopy of the classical groups, Ann. of Math. 70 (1959), 313-337.

[6] M. Golasiński and D.L. Gonçalves, Postnikov towers and Gottlieb groups of orbit spaces, Pacific J. Math. 197, no. 2 (2001), 291-300. 
[7] D. Gottlieb, A certain subgroup of the fundamental group, Amer. J. of Math. 87 (1965), 840-856.

[8] - Evaluation subgroups of homotopy groups, Amer. J. of Math. 91 (1969), 729-756.

[9] - On fibre spaces and the evaluation map, Ann. of Math. 87 (1968), 42-56; correction: ibid. 91 (1970), 640-642.

[10] P.J. Hilton, A note on the P-homomorphism in homotopy groups of spheres, Proc. Camb. Phil. Soc. 59 (1955), 230-233.

[11] P.J. Hilton and J.H.C. Whitehead, Note on the Whitehead product, Ann. of Math. 58 (1953), 429-442.

[12] C.S. Hoo and M. Mahowald, Some homotopy groups of Stiefel manifolds, Bull. Amer. Math. Soc. 71 (1965), 661-667.

[13] H. Kachi and J. Mukai, Some homotopy groups of rotation groups $R_{n}$, Hiroshima J. Math. 29 (1999), 327-345.

[14] M. Kervaire, Some nonstable homotopy groups of Lie groups, Illinois J. Math. 4 (1960), 161-169.

[15] L. Kristensen and I. Madsen, Note on Whitehead products of spheres, Math. Scand. 21 (1967), 301-314.

[16] G.E. Lang, Localizations and evaluation subgroups, Proc. Amer. Math. Soc. 5 (1975), 489-494.

[17] A. Liulevicius, "The factorization of cyclic reduced powers by secondary cohomology operations", Mem. Amer. Math. Soc. 42 (1962).

[18] M. Mahowald, Some Whitehead products in $\mathbb{S}^{n}$, Topology 4 (1965), 1726.

[19] _ "The metastable homotopy of $\mathbb{S}^{n}$ ", Mem. Amer. Math. Soc. 72 (1967).

[20] - A new infinite family in ${ }_{2} \pi_{*}^{s}$, Topology 16 (1977), 249-256.

[21]—, Private communication. 
[22] M. Mimura, On the generalized Hopf homomorphism and the higher composition, Part I; Part II. $\pi_{n+i}\left(\mathbb{S}^{n}\right)$ for $i=21$ and 22, J. Math. Kyoto Univ. 4 (1964), 171-190; 4 (1965), 301-326.

[23] M. Mimura, M. Mori and N. Oda, Determination of 2-components of the 23 and 24-stems in homotoy groups of spheres, Fac. Sci. Kyushu Univ. A 29 (1975), 1-42.

[24] M. Mimura and H. Toda, The $(n+20)$-th homotopy groups of $n$-sphere, J. Math. Kyoto Univ. 3 (1963), 37-58.

[25] Y. Nomura, Note on some Whitehead products, Proc. Japan Acad. 50 (1974), 48-52.

[26] - On the desuspension of Whitehead products, J. London Math. Soc. (2) 22 (1980), 374-384.

[27] N. Oda, Hopf invariants in metastable homotoy groups of spheres, Mem. Fac. Sci. Kyushu Univ. A 30(1976), 221-246.

[28] — , "Unstable homotopy groups of spheres", Bull. Inst. Advanced Research, Fukuoka Univ. no. 44 (1979), 49-152.

[29] J.-P. Serre, Groupes d'homotopie et classes groupes abéliens, Ann. of Math. 58 (1953), 258-294.

[30] S. Thomeier, Einige Ergebnisse über Homotopiegruppen von Sphären, Math. Ann. 164 (1966), 225-250.

[31] H. Toda, "Composition methods in homotopy groups of spheres", Ann. of Math. Studies 49, Princeton (1962).

[32] - Order of the identity class of a suspension space, Ann. of Math. 78 (1963), 300-325.

[33] - On iterated suspensions, I; II, J. Math. Kyoto Univ. 5(1965), 87-142; 5(1966), 209-250.

[34] — "Unstable 3-primary homotopy groups of spheres", Study of Econoinformatics no. 29, Himeji Dokkyo Univ. (2003).

[35] , Private communication. 
[36] G.W. Whitehead, A generalization of the Hopf invariant, Ann. of Math. 51(1950), 192-237.

[37] — , "Elements of homotopy theory", Springer-Verlag, Berlin (1978).

FaCUlty of Mathematics

And Computer Science

NiCholas COPERNICUS UNIVERSITY

87-100 Toruń, Chopina 12/18

POLAND

e-mail: marek@mat.uni.torun.pl

Department of Mathematical Sciences

Faculty of Science, Shinshu University

MATSUMOTO 390-8621, JAPAN

e-mail: jmukai0@gipac.shinshu-u.ac.jp 